\title{
Cryo-EM structure of an activated GPCR-G protein complex in lipid nanodiscs
}

Meng Zhang ${ }^{1,5}$, Miao Gui ${ }^{1,5}$, Zi-Fu Wang ${ }^{1}$, Christoph Gorgulla ${ }^{1}$, James J Yu ${ }^{1}$, Hao Wu ${ }^{1}$, Zhen-yu Sun $^{1}$, Christoph Klenk ${ }^{3}$, Lisa Merklinger ${ }^{3}$, Lena Morstein ${ }^{3}$, Franz Hagn², Andreas Plückthun ${ }^{3}$, Alan Brown ${ }^{1 *}$, Mahmoud L Nasr ${ }^{4 *}$, Gerhard Wagner ${ }^{1 *}$.

\section{Affiliations:}

${ }^{1}$ Department of Biological Chemistry and Molecular Pharmacology, Blavatnik Institute, Harvard Medical School, Boston, MA, USA.

${ }^{2}$ Center for Integrated Protein Science Munich (CIPSM) at the Department of Chemistry and Institute for Advanced Study, Technical University of Munich, Garching, Germany

${ }^{3}$ Department of Biochemistry, University of Zurich, Zurich, Switzerland

${ }^{4}$ Department of Medicine, Brigham and Women's Hospital, Harvard Medical School, Boston, MA, USA

${ }^{5}$ These authors contributed equally: Meng Zhang, Miao Gui

*Corresponding author. E-mail: Alan_Brown@hms.harvard.edu (A.B.);

mnasr@bwh.harvard.edu (M.L.N.); Gerhard_Wagner@hms.harvard.edu (G.W.). 
Abstract: G protein coupled receptors (GPCRs) are the largest superfamily of transmembrane proteins and the targets of over $30 \%$ of currently marketed pharmaceuticals ${ }^{1,2}$. Although several structures have been solved for GPCR-G protein complexes $^{3-17}$, structural studies of the complex in a physiological lipid membrane environment are lacking. Additionally, most previous studies required additional antibodies/nanobodies and/or engineered $\mathrm{G}$ proteins for complex stabilization. In the absence of a native complex structure, the underlying mechanism of $\mathrm{G}$ protein activation leading to GDP/GTP exchange remains unclear. Here, we report cryo-EM structures of lipid bilayer-bound complexes of neurotensin, neurotensin receptor 1 , and $G \alpha_{i 1} \beta_{1} \gamma_{1}$ protein in two conformational states, resolved to 4.1 and $4.2 \AA$ resolution. The structures were determined without any stabilizing antibodies/nanobodies, and thus provide a native-like platform for understanding the structural basis of GPCR-G protein complex formation. Our structures reveal an extended network of protein-protein interactions at the GPCR-G protein interface compared to in detergent micelles, defining roles for the lipid membrane in modulating the structure and dynamics of complex formation, and providing a molecular explanation for the stronger interaction between GPCR and G protein in lipid bilayers. We propose a detailed allosteric mechanism for GDP release, providing new insights into the activation of $\mathrm{G}$ proteins for downstream signaling under near native conditions. 
G protein coupled receptors (GPCRs) sense extracellular stimuli including odorants, hormones, neurotransmitters, and photons. A stimulus leads to a shift in the conformational equilibrium of the GPCR towards a state which favors binding of the intracellular signal transducer, GDP-bound heterotrimeric G $\alpha \beta \gamma \operatorname{protein}^{18}$. Binding causes perturbation of the GDP binding pocket, leading to

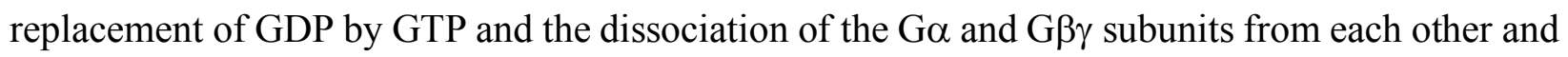
the GPCR ${ }^{19}$. The released G $\alpha$ and G $\beta \gamma$ subunits remain anchored to the membrane through lipid modifications but diffuse and interact with downstream effectors to stimulate signaling cascades ${ }^{18}$.

Recent advances in X-ray crystallography and cryo-EM have allowed the determination of several GPCR-G protein complex structures to near-atomic resolution ${ }^{3-8,11-17,20}$. However, due to difficulties in preparing stable GPCR-G protein complexes in detergent micelles, a range of stabilization techniques had to be employed that compromise the function of the complexes. These

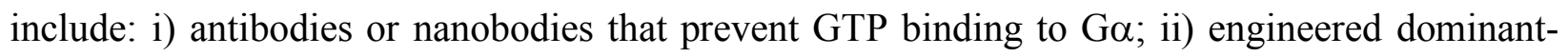
negative G $\alpha$ (DNG $\alpha$ ) subunits that do not exhibit GDP/GTP exchange activity; or iii) engineered mini-G proteins that lack the $\alpha$-helical domain (AHD) of Ga. Furthermore, all the previous structural studies reconstituted GPCR-G protein complexes in detergent micelles, which fail to replicate the properties of the native lipid bilayer environment of GPCRs, including membrane thickness, lateral pressure, and curvature ${ }^{21}$. It has been reported that various GPCRs exhibit higher stability and better functionality when incorporated into lipid bilayers as compared to detergent micelles ${ }^{22,23}$. Additionally, negatively charged lipids have been found to allosterically modulate GPCR activation and its selective interaction with $G$ proteins ${ }^{24-26}$. Therefore, structural and dynamical information for the GPCR-G protein interaction in a lipid bilayer environment is necessary to understand the GPCR signal transduction mechanism. 
To investigate the interaction between GPCR and G proteins in lipid bilayers, we used the neurotensin receptor 1 (NTR1)- $\mathrm{G}_{\mathrm{i}}$ interaction as a model system. NTR1 is a class A GPCR that responds to neurotensin (NT), a 13-residue peptide implicated in the pathogenesis of schizophrenia, antinociception, hypothermia, Parkinson's disease and tumor growth ${ }^{1,27}$. To reconstitute and determine the structure of the $\mathrm{NT}_{8-13}-\mathrm{NTR} 1-\mathrm{G} \alpha_{\mathrm{i} 1} \beta_{1} \gamma_{1}$ complex in a lipid bilayer environment we used circularized nanodiscs (cNDs) prepared with covalently circularized membrane scaffold proteins $^{28}$. While structures of the GDP-bound G protein heterotrimer ${ }^{29}$ and GPCR-G protein complexes were so far only available in detergent micelles, such as the recent cryo-EM structure of the NTR1-G $\alpha_{i 1} \beta_{1} \gamma_{2}$ complex stabilized by an antibody (scFv16) and in complex with a pseudopeptide analog of $\mathrm{NT}^{8}$, the data shown here provide insights into the mechanism by which a $\mathrm{G}$ protein is activated by the interaction with GPCR in a lipid bilayer.

\section{Lipid bilayers enhance the efficiency of NT-NTR1-G $\alpha_{i 1} \beta_{1} \gamma_{1}$ complex formation}

To enable efficient expression of NTR1 for purification and structural studies, we took advantage of the TM86V-L167R $\triangle \mathrm{IC} 3 \mathrm{~B}$ construct $^{30}$. Among all NTR1 evolved variants, TM86V-L167R $\triangle \mathrm{IC} 3 \mathrm{~B}$ shows most wild-type-like GDP/GTP exchange activities ${ }^{30}$ and exhibits similar downstream signaling functionality to wild-type NTR1 as measured by the production of inositol1-phosphate (IP1), the final metabolite of the inositol phosphate cascade, with a EC50 of $2.7 \mathrm{nM}$ for wild-type NTR1 and $0.22 \mathrm{nM}$ for TM86V-L167R $\triangle \mathrm{IC} 3 \mathrm{~B}$ (Extended Data Fig. 1a, left). Interestingly, a single mutation of $\mathrm{R} 167^{3.50} \mathrm{~L}$ (superscripts denote Ballesteros-Weinstein numbering ${ }^{31}$ ) completely quenched IP1 production (Extended Data Fig. 1a, right). As we discuss later in details, R1673.50 is involved in direct interaction with $\mathrm{G}_{\mathrm{i}}$ in the complex structures 
determined in this study, explaining partially the critical impact of this residue in the signaling process.

NTR1 was affinity purified using immobilized $\mathrm{NT}_{8-13}$, which ensured selection of properly folded NTR1 only. The purified NT-NTR1 complex was then incorporated into 9-nm diameter covalently circularized nanodiscs (cNDs), containing a mixture of zwitterionic lipid POPC and negatively charged lipid POPG, and belted by circularized membrane scaffold protein cNW9 $\left(\right.$ ref. $^{28}$ ) (Fig. 1a and Extended Data Fig. 1). Heat-treating the purified nanodiscs at $42{ }^{\circ} \mathrm{C}$ for 24 hours improved sample homogeneity (Extended Data Fig. 1c). Circular dichroism measurements showed increased thermostability of NTR1 in cNDs as compared to in detergent micelles, with a transition temperature about $18^{\circ} \mathrm{C}$ higher (Fig. 1b and Extended Data Fig. 2a, b), implying different dynamic properties in lipid bilayer relative to in detergents. The sample was stable at 45 ${ }^{\circ} \mathrm{C}$ for at least 15 days, showing well dispersed and reproducible peaks on two-dimensional nuclear magnetic resonance (2D NMR) spectra (Extended Data Fig. 3a). These observations agree with studies showing that GPCRs are more stable in membrane environments ${ }^{32}$. When Go $\alpha_{11} \beta_{1} \gamma_{1}$ was incorporated into cNDs using the same method, its thermostability also improved relative to in detergent micelles (Extended Data Fig. 2d-f).

To reconstitute the physiological signaling complex, we incubated NT-NTR1-cND with wildtype heterotrimeric human $\mathrm{G \alpha}_{\mathrm{i} 1} \beta_{1} \gamma_{1}$, which is myristoylated on $\mathrm{G} \alpha_{\mathrm{i} 1}$ and prenylated on $\mathrm{G} \gamma_{1}$ (Extended Data Fig. 1d). The NT-NTR1-G $\alpha_{i 1} \beta_{1} \gamma_{1}$ complex in cNDs exhibits high thermostability (Fig. $1 \mathrm{~b}$ and Extended Data Fig. 2c), and the binding affinity of NTR1 to Go $\alpha_{i 1} \beta_{1} \gamma_{1}$ is higher in cNDs than in detergent micelles ( $\mathrm{K}_{\mathrm{D}}$ of $76 \mathrm{nM}$ compared to $1.4 \mu \mathrm{M}$ ) (Fig. 1c), reflecting the essential role the membrane plays in efficient GPCR-G protein complex formation. Further binding kinetic measurements revealed two binding modes in $\mathrm{cNDs}$ with $\mathrm{K}_{\mathrm{D}}$ of $5.8 \mathrm{nM}$ and $38 \mathrm{nM}$, 
respectively (Fig. 1d and Extended Data Fig. 4a, b). The complex in cND is capable of GDP/GTP exchange, as shown by a much higher dissociation rate upon addition of GTP $\gamma \mathrm{S}$ (Extended Data Fig. 4c). However, for the following structural studies, we used apyrase to hydrolyze free GDP, which stabilizes the NT-NTR1-G $\alpha_{\mathrm{i} 1} \beta_{1} \gamma_{1}$ complex.

\section{Cryo-EM structure of the NT-NTR1-G $\alpha_{i 1} \beta_{1} \gamma_{1}$ complex in cNDs}

The higher affinity and improved thermostability of the NT-NTR1-G $\alpha_{i 1} \beta_{1} \gamma_{1}$ complex in lipid bilayers relative to in detergent micelles allowed us to collect cryo-EM data (Fig. 2, Extended Data Fig. 5) for the complexes without the need for further stabilization by antibodies/nanobodies or engineered G proteins. Two-dimensional class averages showed intact complexes within cNDs with uniform 9-nm diameters (Extended Data Fig. 5). Three-dimensional classification of these projections revealed two well-resolved classes, corresponding to "canonical" (C) and "noncanonical" (NC) states of the NT-NTR1-G $\alpha_{i 1} \beta_{1} \gamma_{1}$ complex, at 4.3 and $4.5 \AA$ resolution, respectively (Extended Data Fig. 5). Two conformational states were also seen in the recent cryoEM study of the scFv16-stabilized NTR1-G $\alpha_{i 1} \beta_{1} \gamma_{2}$ complex in detergent micelles $^{8}$, but, as we describe below, these states are different from those that we observe (Fig. 2c). Additional density surrounds NTR1, corresponding to the cNW9 membrane scaffold protein and the lipid bilayer it encloses. Masking out these densities improved the resolutions of the C and NC states to $4.1 \AA$ and $4.2 \AA$ respectively (Extended Data Fig. 5). In these maps, the pitch of helices and many sidechains are clearly resolved (Extended Data Fig. 6), allowing us to confidently place and remodel known atomic models of NT, NTR $1^{30}$ and $G \alpha_{i 1} \beta_{1} \gamma_{1}$ (ref. ${ }^{9,30}$ ). The density of NT is well revolved in both conformations (Extended Data Fig. 6), and adopts similar structure and 
interactions to those observed in detergent micelles ${ }^{8,33}$. The N-terminal helices of G $\beta$ and G $\gamma$ both show weak densities, presumably due to flexibility.

Compared to most reported structures ${ }^{3,4,6,8,11-14,16,17}$, the $\alpha$-helical domain (AHD) of Go $\alpha_{i 1}$ is clearly resolved in both states (Fig. 2a, Extended Data Fig. 7a, b). In the few structures that do report the position of the $\mathrm{AHD}^{5,15,20}$, the position may be affected by crystal contacts and/or the nanobodies/antibodies that were included for stabilization (Extended Data Fig. 7c-f). Our structures lack these constraints and therefore more closely reflect the native orientation and localization of the AHD in the nucleotide-free state. In comparison to the crystal structure of the GDP-bound $\mathrm{G}_{\mathrm{i}}$ trimer $^{29}$, the AHD moves away from its close association with the Ras-like domain of $\mathrm{G} \alpha$ and interacts with the outer strands of the second and third $\beta$ blades of G $\beta$ after GDP release (Fig. 2b, Extended Data Fig. 7a-c). As we discuss later, the large-scale movement of AHD is an important step in the GDP release pathway.

\section{Lipid bilayer modulates GPCR-G protein interaction}

The NT-NTR1-G $\alpha_{i 1} \beta_{1} \gamma_{1}$ complex shows clear interactions with the lipid bilayer in both the C and NC states (Fig. 3a, Extended data Fig. 8). Density at the beginning of the $\alpha$ N-helix of Ga is observed protruding into the lipid bilayer, which corresponds to the myristoylation site of the Ga (Fig. 3b, top panel). Similar density at the C-terminus of $\mathrm{G} \gamma$ corresponds to the prenylation site (Fig. 3b, top panel). These lipid moieties anchor the $\mathrm{G}$ protein to the membrane. Lipid density is also observed above the positively charged $\alpha$ N-helix of G $\alpha$ (Fig. 3b, bottom panel). The sidechains of arginine and lysine residues within this helix are oriented towards the membrane and likely form electrostatic interactions with the negatively charged lipid POPG (Fig. 3b, bottom panel). This observation agrees with the finding that negatively charged lipids strengthen the interaction 
between NTR1 and $\mathrm{G}$ protein ${ }^{26}$. In comparison with other structures of class A GPCR-G complexes, the $\alpha \mathrm{N}$-helices of $\mathrm{C}$ and $\mathrm{NC}$ states solved here are located close to the membrane, while they bend away in structures determined in detergent micelles (Fig. 3c). The observed hydrophobic and electrostatic interactions ensure close proximity of $\mathrm{G}_{\mathrm{i}}$ to NTR1, and thus enhance $\mathrm{G}_{\mathrm{i}}$ binding to NTR1, particularly between the $\alpha \mathrm{N}-\beta 1$ hinge of $\mathrm{G}_{\mathrm{i}}$ and ICL2 of NTR1 as described below (Fig. 4a).

As expected, the majority of NTR1 is buried inside the lipid bilayer, including TM1-4 and TM7, the N-terminal half of TM5, and the C-terminal half of TM6. ICL2 and H8 are partially buried at the membrane surface (Extended Data Fig. 8c). To reveal the effects of the lipid bilayer on the GPCR, we compared our structures with the crystal structure of NTR1 (X-rNTR1) ${ }^{33}$ and the cryo-EM structure of NTR1 in the canonical state $(\mathrm{C}-\mathrm{hNTR} 1)^{8}$ (representing structures of agonist-bound NTR1 in detergent in the absence and presence of $\mathrm{G}_{\mathrm{i}}$, respectively). In lipid bilayers, the core of NTR1 is more compact due to an inward movement of the middle of TM6 (Fig. 3d, Extended Data Fig. 9a), whereas X-rNTR1 and C-hNTR1 superpose well with each other (Extended Data Fig. 9b). Compression of TM6 is likely due to lateral pressure from the lipid bilayer, implying modulation of the folding and dynamics of NTR1 by the lipid bilayer. Relative to the detergent structures, ICL2 and the cytoplasmic side of TM7 and H8 show an upward movement, indicative of membrane association (Fig. 3d, Extended Data Fig. 9a). Overall, the increased compaction and better membrane association of NTR1 agrees with the improved thermostability observed in lipid bilayers (Fig. 1b, Extended Data Fig. 2c).

Upon insertion of the $\alpha 5$ helix of G $\alpha$ into the core of NTR1, the cytoplasmic side of TM5, TM6 and ICL3 move outward to accommodate the $\alpha 5$ helix (Fig. 3d). Structural and dynamical changes are also observed in 2D NMR experiments on ${ }^{1} \mathrm{H}^{15} \mathrm{~N}-\mathrm{NTR} 1$ upon binding to $\mathrm{G}_{\mathrm{i}}$ in cNDs 
(Extended Data Fig. 3c). In the presence of the lipid bilayer, this movement appears to be more restricted than the large outward movement observed in detergent, potentially due to the lateral pressure from the lipid bilayer (Fig. 3e). The reduced movement of TM5 and TM6 relative to ChNTR1 maintains closer contacts with the $\alpha 5$ helix (Fig. 3e). Comparison of TM6 positions among class A GPCR-Gi complexes reveals that TM6 in the C-state NTR1 exhibits closest proximity to the $\alpha 5$ helix, likely resulting in more potential interactions (Fig. 3f and Extended Data Fig. 9c). Taken together, these observations suggest that the lipid bilayer constrains the conformation of NTR1 to enhance its interaction with $G_{i}$, agreeing with our observation of higher binding affinity in lipid bilayer (Fig. 1c).

\section{The NTR1-G $\alpha_{i 1} \beta_{1} \gamma_{1}$ interface}

The $\mathrm{C}$ and $\mathrm{NC}$ states are related by a $50^{\circ}$ rotation of $\mathrm{G}_{\mathrm{i}}$ relative to NTR1 (Fig. $4 \mathrm{a}$ ). This change in orientation results in different interactions between the $\alpha \mathrm{N}$ helix and ICL2. In the C state, a potential salt bridge is observed between E28 and R185 4.41 , as well as several potential hydrogen bonds between E28 and S182 $2^{4.38}$, R32 and T179 34.55 , and A31 and K178 ${ }^{34.54}$ (Fig. 4a). In contrast, only one hydrogen bond (between R32 and T178 ${ }^{34.55}$ ) is observed in C-hNTR1 in detergent micelles $^{8}$. These additional contacts with ICL2 in the presence of the lipid bilayer likely result from the closer proximity of the $\alpha \mathrm{N}$ helix to the membrane and NTR1 (Fig. 3c). Many of these interactions are absent in the NC state, where we observe only one potential salt bridge between $\mathrm{E} 28$ and $\mathrm{K} 176^{34.52}$. Fewer contacts in the NC state suggest that it could be a less stable intermediate state before the final C-state complex.

The orientation of the $\alpha 5$ helix relative to NTR1 is also different between the two states, although the depth of insertion is the same (Fig. 4b). In the $\mathrm{C}$ state, several potential hydrogen 
bonds are observed, including C351 with E166 $6^{3.49}$, C351 with R167.50, and N347 with A170.53

(Fig. 4b). The interaction between N347 and A170 3.53 is also observed in C-hNTR1 ${ }^{8}$. E166 $6^{3.49}$ and R167.50 belong to the highly conserved D/ERY motif. R167.50 is found to be essential for downstream signaling (Extended Data Fig. 1a) and has been reported to be critical for GDP/GTP exchange through mutagenesis studies ${ }^{30}$. Examination of a range of class A GPCR-G $\mathrm{G}_{\mathrm{i}}$ structures shows that it is common for $\alpha 5$ insertion to stop at $\mathrm{R}^{3.50}$ (Extended Data Fig. 10d). Thus, $\mathrm{R}^{3.50}$ might serve as both an interaction hot-spot and an "access gate" that decides the depth of $\alpha 5$ insertion. The NC state displays fewer interactions with only one possible hydrogen bond between C351 and R167 3.50 (Fig. 4b).

Rotation of $\mathrm{G}_{\mathrm{i}}$ also results in the $\beta 6 \alpha 4$ loop moving closer to ICL3 in the $\mathrm{C}$ state than in either the NC state (Fig. 4c) or detergent structures (Extended Data Fig. 10b). Although the map quality of ICL3 prevents a detailed analysis, molecular dynamics simulations show potential salt bridges and hydrogen bonds forming between ICL3 and $\beta 6 \alpha 4$ loop in the C state (Extended Data Fig. 11). Similar interactions between ICL3 and the $\beta 6 \alpha 4$ loop have been observed in the structure of the adenosine $\mathrm{A} 1$ receptor $\left(\mathrm{A}_{1} \mathrm{R}\right)-\mathrm{G} \alpha_{\mathrm{i} 2} \beta_{1} \gamma_{2}$ complex $^{12}$.

\section{An insertion-rotation model for $\mathbf{G}_{\mathbf{i}}$ activation}

Comparison of our two conformational states with one another and with previous structures allows us to propose a mechanism of G-protein activation under near-native conditions. The presence of more GPCR-G $\mathrm{G}_{\mathrm{i}}$ contacts in the $\mathrm{C}$ state than the $\mathrm{NC}$ state, suggests that the $\mathrm{NC}$ state might be an intermediate, lower-affinity state. This implies that in addition to the close proximity between GPCR and $\mathrm{G}_{\mathrm{i}}$ regulated by lipid bilayer, a certain orientation of $\mathrm{G}_{\mathrm{i}}$ relative to $\mathrm{GPCR}$ is also required to enable efficient complex formation. This is consistent with our kinetics experiments which 
showed both high $(5.8 \mathrm{nM})$ and lower affinity $(38 \mathrm{nM})$ binding modes (Fig. 1d and Extended Data Fig. 4). A sequential model was also proposed to link the states observed with scFv16-stabilized hNTR1-G $\mathrm{G}_{\mathrm{i}}$ in detergent micelles ${ }^{8}$. Following this hypothesis, it appears that the interaction between NTR1 and $G_{i}$ goes through an insertion-rotation mechanism (Fig. 4i). NTR1 and $G_{i}$ first laterally diffuse in membrane until they encounter with each other. The cavity in NTR1 allows insertion of the $\alpha 5$ helix into the open core of NTR1. Subsequently, $\mathrm{G}_{\mathrm{i}}$ rotates around $\alpha 5$ by approximately $50^{\circ}$, which maximizes protein-protein interactions (Fig. 4, Extended Data Fig. 10). The rotation stops when the $\beta 6 \alpha 4$ loop collides with ICL3, the $\alpha \mathrm{N}-\beta 1$ hinge is caught by ICL2, and the $\alpha 5$ helix forms most contacts with the core of NTR1. As the $\alpha 5$ helix in the NT-NTR1-G $\alpha_{i 1} \beta_{1} \gamma_{1} \mathrm{C}$ state exhibits one of the far-most rotated positions among class A GPCR-G $\mathrm{G}_{\mathrm{i}}$ complexes (Extended Data Fig. 10d), the NT-NTR1-G $\alpha_{i 1} \beta_{1} \gamma_{1} \mathrm{C}$ state likely represents the final state enabling GDP dissociation (Fig. 4i).

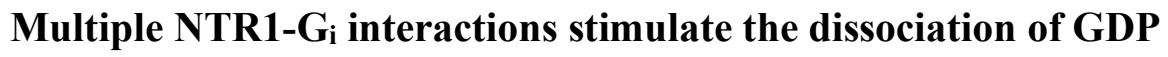

Based on comparison of our structures with the structure of GDP-Gi ${ }^{29}$, we propose a multipartite mechanism for receptor-catalyzed nucleotide exchange (Fig. 5). In the unbound G-protein, the nucleotide is buried between the ras-homology domain (RHD) and the AHD of Ga. It has been suggested that when $G$ protein encounters the receptor, $\alpha 5$ is straightened and forms early interactions with the GPCR, which initiates the GDP release process ${ }^{34}$. The AHD dissociates from the RHD, and, as we show here, interacts with the outermost strands of G $\beta$ (Extended Data Fig. 7a, b). Previous computational simulations have shown that separation of the AHD is necessary (presumably to create an exit pathway for GDP) but not sufficient for rapid nucleotide release ${ }^{35,36}$. Here we observe that multiple allosteric pathways converge on structural rearrangement of the 
GDP binding site, and it is the combination of these pathways that are responsible GDP dissociation.

In the first pathway, insertion and rotation of the $\alpha 5$ helix into the core of NTR1 by two helical turns compared to the GDP-G $\mathrm{G}_{\mathrm{i}}$ structure $^{29}$ displaces the $\alpha 5 \beta 6$ loop, which is responsible for binding the guanine ring of GDP in the nucleotide-bound state (Fig. 4e). This is consistent with previous structural studies showing that the $\alpha 5 \beta 6$ loop perturbation induced by the rotational translation of $\alpha 5$ helix is essential for GDP dissociation. ${ }^{3,4,15-17,20,37,38,5,6,8,9,11-14}$ As a result of this perturbation, A326 in the highly conserved TCAT motif moves away from its position in the GDP$\mathrm{G}_{\mathrm{i}}$ structure resulting in loss of contact with GDP. This agrees with previous mutagenesis studies showing that A326 is essential for GDP binding ${ }^{39}$. The conformation of the $\alpha 5 \beta 6$ loop is different from that in the detergent structure, potentially as a result of the different angles with which $\alpha 5$ inserts into NTR1 (Extended Data Fig. 10c, d). This agrees with computational simulations in which the tilt angle of the $\alpha 5$ helix was found to directly correlate with the conformation of the $\alpha 5 \beta 6$ loop $^{36}$. The new conformation of the $\alpha 5 \beta 6$ loop, and therefore the dynamics of GDP loss, may be affected by the neighboring interaction between ICL3 and the $\beta 6 \alpha 4$ loop (Fig. 4c, e, Extended data Fig. 11).

In the second pathway, displacement of AHD appears to be correlated with movement of $\alpha 1$ (to which AHD is tethered) (Fig. 4f, g). This lateral movement causes residues within $\alpha 1$, including S47, to dissociate from the phosphate group of GDP (Fig. 4f, g). The S47N mutation is dominant negative ${ }^{14}$, suggesting that this movement is a key step towards GDP release. Our observation here agrees with previous mutagenesis ${ }^{40}, \mathrm{HDX}^{37}$ as well as computational ${ }^{41}$ studies that suggested perturbations in $\alpha 1$ play important roles in accelerating GDP dissociation. 
In the third pathway, the interaction between ICL2 of NTR1 and the $\alpha \mathrm{N}-\beta 1$ hinge propagates through the $\beta 1$ strand and perturbs the GDP phosphate-binding P-loop ( $\alpha 1 \beta 1$ loop) (Fig. 4d). Such a P-loop perturbation by the $\alpha$ N $\beta 1$-ICL2 interaction is also supported by previous structural ${ }^{3,4,20}$ and $\mathrm{HDX}^{37}$ studies. This perturbation results in a displacement of the P-loop including the mainchain of residue A41 so that it would no longer be able to make direct interactions with the $\beta$-phosphate of GDP (Fig. 4d). To accommodate the displaced P loop, the sidechain of E245 on $\alpha 2$ has rotated by $95^{\circ}$ (Fig. 4h). This implies a coupling of P-loop disorder with E245 rotation in the GDP dissociation process, and conversely a role for E245 in maintaining a stable GDP-bound G protein conformation, which coincides with the E245A mutant causing dominant negative effects suggesting a key role of the side chain of E245 in the GDP/GTP exchange process ${ }^{12,39}$. This rotation is not observed in the detergent-embedded NTR1-G $\mathrm{i}_{\mathrm{i}}$ structure, as the P loop adopts a conformation more similar to the one observed in the GDP-G $\mathrm{G}_{\mathrm{i}}$ structure ${ }^{29}$ (Fig. 4h).

Together, this multi-point coordination mechanism leads to complete dissociation of GDP from $\mathrm{G}_{\mathrm{i}}$ and the creation of a free nucleotide binding pocket for GTP association (Fig. 5).

\section{Conclusion}

Understanding the structural basis for the interaction between GPCRs and G proteins under physiological conditions has been challenging due to the poor stability of the complexes in detergent micelles. Most of the published structures required antibodies/nanobodies and/or engineered $G$ proteins for additional stability, which rendered the complexes incapable of GDP/GTP exchange. Using our recently developed covalently circularized nanodiscs ${ }^{28}$, we have determined two structures, representing different conformational states, of the NT-NTR1-G $\alpha_{i 1} \beta_{1} \gamma_{1}$ 
complex in a lipid bilayer without the need for external stabilization. These structures reflect the native signaling-competent states of the complex. They show that the sideway movement of TM6, which is considered a signature of active receptors in detergents, is restricted here by the membrane, highlighting the importance of the membrane in modulating the dynamics of GPCR-G protein interactions and the affinity between NTR1 with $\mathrm{G}_{\mathrm{i}}$. Our structures also allowed us to unravel the interconnected roles of membrane-protein interaction, G-protein activation, and GDP dissociation. The proposed multipartite allosteric mechanism of GDP release reveals a competition between GDP and NTR1 for binding $\mathrm{G}_{\mathrm{i}}$, which explains why the NTR1-G $\mathrm{G}_{\mathrm{i}}$ complex is stabilized by removal of GDP using apyrase. This observation agrees with a previous NMR study showing that the interaction between NTR1 and G $\alpha$ is strongest when G $\alpha$ is nucleotide free ${ }^{42}$. Our study therefore provides new insights into the signal transduction process triggered by GPCR-G protein complex formation and will serve as a model for future studies of GPCR signaling in native lipid bilayers.

\section{References}

1. Griebel, G. \& Holsboer, F. Neuropeptide receptor ligands as drugs for psychiatric diseases: The end of the beginning? Nature Reviews Drug Discovery 11, 462-478 (2012).

2. Shimada, I., Ueda, T., Kofuku, Y., Eddy, M. T. \& Wüthrich, K. GPCR drug discovery: Integrating solution NMR data with crystal and cryo-EM structures. Nature Reviews Drug Discovery 18, 59-82 (2018).

3. Zhang, Y. et al. Cryo-EM structure of the activated GLP-1 receptor in complex with a G protein. Nature 546, 248-253 (2017).

4. Liang, Y. L. et al. Phase-plate cryo-EM structure of a class B GPCR-G-protein complex. Nature 546, 118-123 (2017). 
5. Qi, X. et al. Cryo-EM structure of oxysterol-bound human Smoothened coupled to a heterotrimeric Gi. Nature (2019). doi:10.1038/s41586-019-1286-0

6. Krishna Kumar, K. et al. Structure of a Signaling Cannabinoid Receptor 1-G Protein Complex. Cell 176, 448-458.e12 (2019).

7. Zhao, L.-H. et al. Structure and dynamics of the active human parathyroid hormone receptor-1. Science (80-. ). 364, 148-153 (2019).

8. Kato, H. E. et al. Conformational transitions of a neurotensin receptor 1-Gi1 complex. Nature 572, 80-85 (2019).

9. Gao, Y. et al. Structures of the Rhodopsin-Transducin Complex: Insights into G-Protein Activation. Mol. Cell 75, 781-790.e3 (2019).

10. Tsai, C. J. et al. Crystal structure of rhodopsin in complex with a mini-Go sheds light on the principles of G protein selectivity. Sci. $A d v .4$, (2018).

11. García-Nafría, J., Nehmé, R., Edwards, P. C. \& Tate, C. G. Cryo-EM structure of the serotonin 5-HT1B receptor coupled to heterotrimeric Go. Nature 558, 620-623 (2018).

12. Draper-Joyce, C. J. et al. Structure of the adenosine-bound human adenosine A1 receptorGi complex. Nature 558, 559-563 (2018).

13. Liang, Y. L. et al. Cryo-EM structure of the active, G s -protein complexed, human CGRP receptor. Nature 561, 492-497 (2018).

14. Liang, Y. L. et al. Phase-plate cryo-EM structure of a biased agonistbound human GLP-1 receptor-Gs complex. Nature 555, 121-125 (2018).

15. Kang, Y. et al. Cryo-EM structure of human rhodopsin bound to an inhibitory G protein. 
Nature 558, 553-558 (2018).

16. Koehl, A. et al. Structure of the $\mu$-opioid receptor-G i protein complex. Nature 558, 547552 (2018).

17. García-Nafría, J., Lee, Y., Bai, X., Carpenter, B. \& Tate, C. G. Cryo-EM structure of the adenosine A2A receptor coupled to an engineered heterotrimeric G protein. Elife 7, (2018).

18. Hilger, D., Masureel, M. \& Kobilka, B. K. Structure and dynamics of GPCR signaling complexes. Nat. Struct. Mol. Biol. 25, 4-12 (2018).

19. Du, Y. et al. Assembly of a GPCR-G Protein Complex. Cell 177, (2019).

20. Rasmussen, S. G. F. et al. Crystal structure of the $\beta 2$ adrenergic receptor-Gs protein complex. Nature 477, 549-557 (2011).

21. Lee, A. G. How lipids affect the activities of integral membrane proteins. Biochimica et Biophysica Acta - Biomembranes 1666, 62-87 (2004).

22. Whorton, M. R. et al. Efficient coupling of transducin to monomeric rhodopsin in a phospholipid bilayer. J. Biol. Chem. 283, 4387-94 (2008).

23. Kofuku, Y. et al. Functional dynamics of deuterated $\beta 2$-adrenergic receptor in lipid bilayers revealed by NMR spectroscopy. Angew. Chemie - Int. Ed. 53, 13376-13379 (2014).

24. Strohman, M. J. et al. Local membrane charge regulates $\beta 2$ adrenergic receptor coupling to G i3. Nat. Commun. 10, (2019).

25. Yen, H.-Y. et al. PtdIns(4,5)P2 stabilizes active states of GPCRs and enhances selectivity 
of G-protein coupling. Nature 559, 423-427 (2018).

26. Inagaki, S. et al. Modulation of the Interaction between Neurotensin Receptor NTS1 and Gq Protein by Lipid. J. Mol. Biol. 417, 95-111 (2012).

27. Kitabgi, P. Targeting neurotensin receptors with agonists and antagonists for therapeutic purposes. Curr. Opin. Drug Discov. Devel. 5, 764-76 (2002).

28. Nasr, M. L. et al. Covalently circularized nanodiscs for studying membrane proteins and viral entry. Nat. Methods 14, 49-52 (2016).

29. Wall, M. A. et al. The structure of the G protein heterotrimer Gi alpha 1 beta 1 gamma 2 . Cell 83, 1047-58 (1995).

30. Egloff, P. et al. Structure of signaling-competent neurotensin receptor 1 obtained by directed evolution in Escherichia coli. Proc. Natl. Acad. Sci. 111, E655-E662 (2014).

31. Ballesteros, J. A. \& Weinstein, H. Integrated methods for the construction of threedimensional models and computational probing of structure-function relations in $\mathrm{G}$ protein-coupled receptors. Methods Neurosci. 25, 366-428 (1995).

32. Knepp, A. M., Grunbeck, A., Banerjee, S., Sakmar, T. P. \& Huber, T. Direct Measurement of Thermal Stability of Expressed CCR5 and Stabilization by Small Molecule Ligands. Biochemistry 50, 502-511 (2011).

33. Krumm, B. E., White, J. F., Shah, P. \& Grisshammer, R. Structural prerequisites for Gprotein activation by the neurotensin receptor. Nat. Commun. 6, 7895 (2015).

34. Liu, X. et al. Structural Insights into the Process of GPCR-G Protein Complex Formation. Cell 177, 1243-1251.e12(2019). 
35. Dror, R. O. et al. Structural basis for nucleotide exchange in heterotrimeric G proteins. Science (80-. ). 348, 1361-1365 (2015).

36. Sun, X., Singh, S., Blumer, K. J. \& Bowman, G. R. Simulation of spontaneous G protein activation reveals a new intermediate driving GDP unbinding. Elife 7, (2018).

37. Chung, K. Y. et al. Conformational changes in the G protein Gs induced by the $\beta 2$ adrenergic receptor. Nature 477, 611-617 (2011).

38. Erlandson, S. C., McMahon, C. \& Kruse, A. C. Structural Basis for G Protein-Coupled Receptor Signaling. Annu. Rev. Biophys. 47, 1-18 (2018).

39. Iiri, T., Bell, S. M., Baranski, T. J., Fujita, T. \& Bourne, H. R. A Gs $\alpha$ mutant designed to inhibit receptor signaling through Gs. Proc. Natl. Acad. Sci. U. S. A. 96, 499-504 (1999).

40. Sun, D. et al. Probing Ga i1 protein activation at single-amino acid resolution. Nat. Struct. Mol. Biol. 22, 686-694 (2015).

41. Flock, T. et al. Universal allosteric mechanism for Ga activation by GPCRs. Nature 524, $173-179$ (2015).

42. Goricanec, D. et al. Conformational dynamics of a G-protein $\alpha$ subunit is tightly regulated by nucleotide binding. Proc. Natl. Acad. Sci. 113, E3629-E3638 (2016).

\section{Methods}

No statistical methods were used to predetermine sample size. The experiments were not randomized and the investigators were not blinded to allocation during experiments and outcome assessment.

\section{Preparation of NTR1 in cNDs}


Expression and purification of a thermostable variant of rat NTR1 (TM86V-L167R $\Delta$ IC3B) was performed as described previously with some modifications ${ }^{30,43}$. This NTR1 variant consists of residues G50-G390, contains a deletion of E273-T290 in intracellular loop (ICL) 3, and has ten stabilizing mutations. Briefly, the full-length fusion protein consisting of maltose-binding protein (MBP), NTR1, and thioredoxin (TrxA) was expressed in Tuner ${ }^{\mathrm{TM}}$ (DE3) Competent Cells (Novagen) in LB medium at $37^{\circ} \mathrm{C}, 200 \mathrm{rpm}$ and induced at an $\mathrm{OD}_{600}$ of 0.75 with $1 \mathrm{mM}$ IPTG. Cells were grown for another 24 hours at $20{ }^{\circ} \mathrm{C}, 160 \mathrm{rpm}$ and harvested by centrifugation $(5,000$ $\times g, 30 \mathrm{~min}, 4^{\circ} \mathrm{C}$ ). Cells were then lysed and solubilized by sonication in buffer containing 100 $\mathrm{mM}$ HEPES (pH 8.0), 20\% glycerol, $400 \mathrm{mM} \mathrm{NaCl}, \quad 2.5 \mathrm{mM} \mathrm{MgCl}_{2}, \quad 0.6 / 0.12 \%$ CHAPS/cholesterol, 1.7\% n-decyl- $\beta$-D-maltopyranoside (DM), $100 \mathrm{mg}$ lysozyme, one tablet of protease inhibitor, and $250 \mathrm{U}$ benzonase. Cell lysate was centrifuged, and the supernatant was mixed with pD-NT resin ${ }^{43}$ pre-equilibrated with 25 mM HEPES (pH 8.0), 10\% glycerol, $600 \mathrm{mM}$ $\mathrm{NaCl}$ and $0.5 \% \mathrm{DM}$ at $4{ }^{\circ} \mathrm{C}$ for 1 hour. The flow-through from the $\mathrm{pD}-\mathrm{NT}$ resin was then discarded, and the resin was washed with $25 \mathrm{mM}$ HEPES (pH 7.0), 10\% glycerol, $150 \mathrm{mM} \mathrm{NaCl}, 2 \mathrm{mM}$ DTT and $0.3 \% \mathrm{DM}$. The resin was then mixed with $3 \mathrm{C}$ protease for 1 hour at $4{ }^{\circ} \mathrm{C}$ to cleave off $\mathrm{MBP}$ and TrxA from NTR1, as well as NT-NTR1 from $\mathrm{pD}$ resin ${ }^{43}$. The resin was washed with $10 \mathrm{mM}$ HEPES (pH 7.0), 10\% glycerol, 2 mM DTT and 0.3\% DM, which was combined with the flowthrough and loaded onto a SP cation exchange chromatography column (GE Healthcare) preequilibrated in the same washing buffer. The SP column was washed with $10 \mathrm{mM}$ HEPES (pH 7.7), 10\% glycerol, $35 \mathrm{mM} \mathrm{NaCl}, 2 \mathrm{mM}$ DTT and 1\% diheptanoylphosphatidylcholine $\left(\mathrm{DH}_{7} \mathrm{PC}\right)$, and then eluted with $10 \mathrm{mM}$ HEPES (pH 7.7), 10\% glycerol, $350 \mathrm{mM} \mathrm{NaCl,} 2 \mathrm{mM}$ DTT and $0.2 \%$ $\mathrm{DH}_{7} \mathrm{PC}$. The eluate was concentrated to below $500 \mu \mathrm{L}$ and subjected to size-exclusion chromatography on a Superdex 200 10/300 Increase Analytical (S200a) column (GE Healthcare) 
equilibrated with $10 \mathrm{mM}$ HEPES (pH 7.7), $150 \mathrm{mM} \mathrm{NaCl}, 2 \mathrm{mM}$ DTT, $0.1 \% \mathrm{DH}_{7} \mathrm{PC}$ and $0.1 \mu \mathrm{M}$ NT. Fractions containing NTR1 were collected and mixed with a 3:2 molar ratio of 1-Palmitoyl2-oleoyl-phosphatidylcholine (POPC) to 1-palmitoyl-2-oleoyl-phosphatidylglycerol (POPG) solubilized in $100 \mathrm{mM}$ sodium cholate at a NTR1:lipid molar ratio of 1:160. The mixture was incubated on ice for 30 min before addition of cNW9 at a cNW9:NTR1 molar ratio of 4:1 followed by another $30 \mathrm{~min}$ incubation on ice. The mixture was then treated with 5\% volume of Bio-Bead SM-2 resin (Bio-Rad) with shaking on ice for $15 \mathrm{~min}$, followed by addition of another $20 \%$ volume of Bio-Beads every 20 min for detergent removal. After two-hour incubation with Bio-Beads, the flow-through was then subjected to size-exclusion chromatography with a S200a column equilibrated in $20 \mathrm{mM}$ sodium phosphate (pH 6.9), $50 \mathrm{mM} \mathrm{NaCl}, 1 \mathrm{mM}$ DTT, $0.5 \mathrm{mM}$ EDTA, 0.1 $\mu \mathrm{M}$ NT. Fractions containing NTR1-cND were concentrated to below $500 \mu \mathrm{L}$ and incubated at $42{ }^{\circ} \mathrm{C}$ for 24 hours, followed by filtration through $0.22 \mu \mathrm{m}$ cut-off filters. The flow-through was subjected to another round of size-exclusion chromatography. Fractions were pooled, concentrated and stored at $4^{\circ} \mathrm{C}$.

\section{Preparation of $\mathrm{G} \alpha_{\mathrm{i} 1} \beta_{1} \gamma_{1}$ in micelles and $\mathrm{cNDs}$}

G protein composed of $G \alpha_{i 1}, G \beta_{1}$ and $G \gamma_{1}$ was expressed and purified as detailed before ${ }^{30,44}$. Briefly, Spodoptera frugiperda (Sf9) were grown in suspension in ESF921 medium (Expression Systems, California), infected at a density of $2-3 \times 10^{6} / \mathrm{mL}$ with a single baculovirus encoding all three subunits $\left(\mathrm{G} \alpha_{\mathrm{i} 1} \beta_{1} \gamma_{1}\right)$, harvested within 72 hours post inoculation, and stored at $-80{ }^{\circ} \mathrm{C}$ until use. 
Cells were lysed in 10 mM HEPES (pH 7.4), $20 \mathrm{mM} \mathrm{KCl,} 10 \mathrm{mM} \mathrm{MgCl} 2,10 \mu \mathrm{M} \mathrm{GDP}, 2 \mathrm{mM} \beta-$ mercaptoethanol ( $\beta-\mathrm{ME})$, and 1 protease inhibitor tablet with sonication. The suspension was then ultra-centrifuged at $180,000 \times g$ for $45 \mathrm{~min}$ at $4{ }^{\circ} \mathrm{C}$. The membrane pellet was solubilized in 50 mM HEPES (pH 7.4), 150 mM NaCl, 10 mM MgCl $2,10 \mu \mathrm{M}$ GDP, 2 mM $\beta$-ME, 10\% glycerol, 1 protease inhibitor tablet, $1.2 \% \mathrm{DM}$ at $4{ }^{\circ} \mathrm{C}$ for 3 hours. The suspension was ultra-centrifuged again and the supernatant was purified through Ni-NTA resin ${ }^{20}$. The eluate was concentrated and run through a Superdex 200 prep 16/60 column (S200p; GE Healthcare) equilibrated in 20 mM HEPES (pH 7.4), $100 \mathrm{mM} \mathrm{NaCl}, 0.1 \mathrm{mM} \mathrm{MgCl}_{2}, 4 \mathrm{mM} \beta-\mathrm{ME}$, and $0.5 \% \mathrm{DM}$. Fractions containing G $\alpha_{i 1} \beta_{1} \gamma_{1}$ were pooled and concentrated to $10 \mathrm{mg} / \mathrm{mL}$, flash-frozen in liquid nitrogen and stored at $-80{ }^{\circ} \mathrm{C}$.

$\mathrm{G \alpha}_{\mathrm{i} 1} \beta_{1} \gamma_{1}-\mathrm{cNDs}$ were prepared similarly as for NTR1-cNDs. After Bio-Bead removal, the G⿰$\alpha_{i 1} \beta_{1} \gamma_{1-}$ -cNDs were purified through Ni-NTA to remove empty cNDs, followed by S200a chromatography to remove aggregates. Fractions containing pure Go $\alpha_{i 1} \beta_{1} \gamma_{1}$-cNDs were collected, concentrated, and stored at $4^{\circ} \mathrm{C}$.

\section{Complex formation of NT-NTR1-G $\alpha_{i 1} \beta_{1} \gamma_{1}$ in cNDs}

Purified $\mathrm{G \alpha}_{\mathrm{i} 1} \beta_{1} \gamma_{1}$ in micelle was diluted in buffer A (20 mM HEPES (pH 6.9), $50 \mathrm{mM} \mathrm{NaCl}, 5$ $\mathrm{mM} \mathrm{CaCl}_{2}, 1 \mathrm{mM}$ DTT, $0.1 \mu \mathrm{M} \mathrm{NT}$ ) until the DM concentration dropped below $0.08 \%$ (the critical micelle concentration of DM), and mixed with NTR1-cND at 1:1 molar ratio. The mixture was incubated on ice for $30 \mathrm{~min}$, followed by addition of Bio-Beads at $10 \%$ volume every $30 \mathrm{~min}$. The mixture was incubated on ice with shaking for a total of 2 hours and then the Bio-Beads were 
removed. Apyrase, diluted with buffer A and pretreated with Bio-Beads for $30 \mathrm{~min}$ on ice, was added to the complex at $1 \mathrm{U} / \mathrm{mL}$ concentration. The mixture was incubated at $4{ }^{\circ} \mathrm{C}$ overnight, and then subjected to a S200a SEC column equilibrated in $20 \mathrm{mM}$ sodium phosphate (pH 6.9), $50 \mathrm{mM}$ $\mathrm{NaCl}, 1 \mathrm{mM}$ DTT, $0.5 \mathrm{mM}$ EDTA, $0.1 \mu \mathrm{M}$ NT. Peak fractions were characterized with SDSPAGE and negative-stain EM. The fractions containing NT-NTR1-G $\alpha_{i 1} \beta_{1} \gamma_{1}$ in cNDs were used for cryo-EM structure determination.

\section{Circular Dichroism (CD) spectroscopy}

CD spectra were measured on a JASCO J-815 spectropolarimeter equipped with a Peltier cell temperature controller. Both spectrum scan measurement and variable temperature measurement were carried out for the following samples: NTR1 in micelles, NTR1-cNDs, Ga $\alpha_{\mathrm{i} 1} \beta_{1} \gamma_{1}$ in micelles, $\mathrm{G}_{\mathrm{i} 1} \beta_{1} \gamma_{1}-\mathrm{cNDs}$, and NTR1-Go $\alpha_{i 1} \beta_{1} \gamma_{1}$ in cNDs. Spectrum scan measurements were performed at 20 ${ }^{\circ} \mathrm{C}$, before and after variable temperature measurements, collecting data from $260 \mathrm{~nm}$ to $190 \mathrm{~nm}$. Variable temperature measurements were carried out at $220 \mathrm{~nm}$ increasing temperature from 20 ${ }^{\circ} \mathrm{C}$ to $95^{\circ} \mathrm{C}$ at a rate of $1{ }^{\circ} \mathrm{C} / \mathrm{min}$. Spectrum Manager 2 software was used to analyze the transition temperature for each sample.

\section{Binding affinity and kinetics measurement}

Binding affinity and kinetics between NTR1 and $\mathrm{G \alpha}_{\mathrm{i} 1} \beta_{1} \gamma_{1}$ in detergent micelles and cNDs were measured using MicroScale Thermophoresis (MST) and Biolayer Interferometry (BLI). 
For MST, the measurements were performed on a Monolith NT.115 system (NanoTemper Technologies). We measured the fluorescence signal from $G \alpha_{i 1} \beta_{1} \gamma_{1}$ by using the Monolith HisTag Labeling Kit RED-tris-NTA 2nd Generation kit (NanoTemper Technologies). The samples were prepared in a buffer containing $20 \mathrm{mM}$ sodium phosphate $(\mathrm{pH} 6.9), 50 \mathrm{mM} \mathrm{NaCl}, 0.05 \%$ $\mathrm{DH}_{7} \mathrm{PC}$ for $\mathrm{cND}$ titrations and $0.2 \% \mathrm{DH}_{7} \mathrm{PC}$ for titrations in detergent micelle. The concentration of $\mathrm{DH}_{7} \mathrm{PC}$ for $\mathrm{cND}$ titrations is below its critical micellar concentration. The experiments were carried out as fast as possible (within 1-2 minute for sample preparation) to prevent degradation of $\mathrm{G \alpha}_{\mathrm{i} 1} \beta_{1} \gamma_{1}$. The concentration of $\mathrm{G} \alpha_{\mathrm{i} 1} \beta_{1} \gamma_{1}$ was constant at $10 \mathrm{nM}$. NT-NTR1 in $\mathrm{DH}_{7} \mathrm{PC}, \mathrm{NT}-$ NTR1-cND, or empty cND was titrated in two-fold dilution steps beginning at $4 \mu \mathrm{M}$. For the measurement the samples were filled into premium-coated capillaries. The measurement was performed at $2 \%$ LED and $20 \%$ MST power, $30 \mathrm{sec}$ Laser-On, and $5 \mathrm{sec}$ Laser-Off. Fluorescence was excited at $605-645 \mathrm{~nm}$, and emission was detected at 680-685 nm. The results were analyzed using the MO Affinity Analysis software (NanoTemper Technologies). The dissociation constant $\left(K_{D}\right)$ was then determined using a single-site model for data fitting.

BLI experiments were performed on an Octet RED384 (ForteBio, California) using Anti-His antibody-coated Dip and Read Biosensors (HIS1, ForteBio) and 384 well plates (ForteBio) with $60 \mu \mathrm{L}$ volume. $500 \mathrm{nM}$ of His-tagged $\mathrm{G \alpha}_{\mathrm{i} 1} \beta_{1} \gamma_{1}$ was bound for $5 \mathrm{~min}$ in a binding buffer consisting of $20 \mathrm{mM}$ HEPES (pH 7.4), $100 \mathrm{mM} \mathrm{NaCl}, 0.1 \mathrm{mM} \mathrm{MgCl}_{2}, 4 \mathrm{mM} \beta-\mathrm{ME}$, and 0.5\% DM. To test for nonspecific binding of His-tagged $\mathrm{G} \alpha_{i 1} \beta_{1} \gamma_{1}$, reference tips were incubated in buffer alone. The tips were washed with buffer for 2 min to obtain a baseline reading and then transferred to wells in various concentrations of NT-NTR1-cND $(4,2,1,0.5,0.25 \mu \mathrm{M})$ in buffer containing $20 \mathrm{mM}$ sodium phosphate ( $\mathrm{pH} 6.9$ ), $50 \mathrm{mM} \mathrm{NaCl}, 1 \mathrm{mM}$ DTT, $0.5 \mathrm{mM}$ EDTA, $0.1 \mu \mathrm{M}$ NT for $5 \mathrm{~min}$. 
After measuring the association phase, tips were moved to wells containing buffer with and without GTP $\gamma \mathrm{S}$, and dissociation was measured for $5 \mathrm{~min}$. The data were processed and analyzed using the Octet data analysis software version 11.0 (ForteBio). Association-dissociation curves for each concentration were fit to a 2:1 model.

\section{Nuclear Magnetic Resonance (NMR) spectroscopy}

Uniformly ${ }^{15} \mathrm{~N}$-labeled NT-NTR1 in POPC/POPG (3:2) cNW9 nanodiscs at $200 \mu \mathrm{M}$ alone and in complex with unlabeled $\mathrm{G \alpha}_{\mathrm{i} 1} \beta_{1} \gamma_{1}$ at a molar ratio of 5:1 were prepared as described above in NMR buffer (20 mM sodium phosphate ( $\mathrm{pH}$ 6.9), $50 \mathrm{mM} \mathrm{NaCl}, 1 \mathrm{mM}$ DTT, $0.5 \mathrm{mM}$ EDTA, 10\% $\mathrm{D}_{2} \mathrm{O}$ ). Two-dimensional Transverse Relaxation Optimized Spectroscopy (TROSY) Heteronuclear Single Quantum Coherence (HSQC) were collected with 2000 scans, 200 increments at $45^{\circ} \mathrm{C}$ on a Bruker 800-MHz spectrometer equipped with a TXO cryogenic probe. TROSY HSQC measurements were repeated for NT-NTR1-cND on an Agilent 700-MHz spectrometer to verify that NT-NTR1cND stays intact after long data acquisition in the magnet at $45^{\circ} \mathrm{C}$. Data were processed using the NMRPipe software package ${ }^{45}$.

\section{Functional Assay}

Ligand-induced IP1 (a metabolite of IP3) accumulation was measured in transiently transfected HEK293T/17 cells as described before ${ }^{46}$. Wild type rNTR1 or mutants thereof were directly subcloned into a mammalian expression vector containing an N-terminal SNAP-tag (pMC08). 24 hrs after transfection, cells were washed with PBS, detached with Trypsin-EDTA (Sigma) and resuspended in assay buffer (10 mM HEPES pH 7.4, $1 \mathrm{mM} \mathrm{CaCl} 2,0.5 \mathrm{mM} \mathrm{MgCl}_{2}, 4.2 \mathrm{mM} \mathrm{KCl}$, 
$146 \mathrm{mM} \mathrm{NaCl}, 50 \mathrm{mM} \mathrm{LiCl}, 5.5 \mathrm{mM}$ glucose, $0.1 \%$ (w/v) BSA). Cells were seeded at 20,000 cells per well in white 384-well plates (Greiner) and incubated for $2 \mathrm{hrs}$ at $37^{\circ} \mathrm{C}$ with a concentration range of $\mathrm{NT}_{8-13}$ (Anawa) diluted in assay buffer. IP1 accumulation was measured using the HTRF IP-One kit (Cisbio) according to the manufacturer's protocol. To confirm cell surface expression of NTR1 and its mutants, transfected cells were plated on poly-D-lysine treated 384-well plates (Greiner) at 20,000 cells/well in growth medium. The following day, medium was removed and cells were incubated with 50 nM SNAP-Lumi4-Tb (CisBio) in labelling buffer (CisBio) for 2 hrs at $37^{\circ} \mathrm{C}$. Thereafter, cells were washed 4 times with wash buffer $(20 \mathrm{mM}$ HEPES pH 7.5, $100 \mathrm{mM}$ $\mathrm{NaCl}, 3 \mathrm{mM} \mathrm{MgCl} 2$ and $0.2 \%(\mathrm{w} / \mathrm{v})$ nonfat milk). Fluorescence intensity of $\mathrm{Tb}^{3+}$-labelled receptors was measured on an Infinite M1000 fluorescence plate reader (Tecan) with an excitation wavelength of $340 \mathrm{~nm}$ and emission wavelength of $620 \mathrm{~nm}$. To generate concentration-response curves, data were normalized to receptor expression at the cell surface and to response of NTR1 at maximal ligand concentration and were analysed by a non-linear curve fit in GraphPad Prism.

\section{Negative-stain microscopy}

$3 \mu \mathrm{L}$ of NT-NTR1-G $\alpha_{i 1} \beta_{1} \gamma_{1}-\mathrm{cND}$ complex at a concentration of $0.02 \mathrm{mg} / \mathrm{mL}$ was applied onto a glow-discharged continuous carbon grid (Electron Microscopy Sciences, Inc.). After two minutes of adsorption, the grid was blotted with filter paper to remove the excess sample, immediately washed twice with $50 \mu \mathrm{L}$ of MiliQ water, once with $50 \mu \mathrm{L} 0.75 \%$ uranyl formate solution and incubated with $50 \mu \mathrm{L}$ of $0.75 \%$ uranyl formate solution for an additional one minute. The grid was then further blotted with filter paper to remove the uranyl formate solution, air-dried at room temperature, and examined with a Tecnai T12 electron microscope (Thermo Fisher Scientific) 
equipped with an LaB6 filament and operated at 120-kV acceleration voltage, using a nominal magnification of $52,000 \times$ at a pixel size of $2.13 \AA$.

\section{Cryo-EM sample preparation}

Cryo-EM grids were prepared using a Vitrobot Mark IV (Thermo Fisher Scientific). $3 \mu \mathrm{L}$ of NTNTR1-G $\alpha_{i 1} \beta_{1} \gamma_{1}-\mathrm{cND}$ at a concentration between $1.5 \mathrm{mg} / \mathrm{mL}$ to $1.7 \mathrm{mg} / \mathrm{mL}$ was applied onto glow discharged C-flat holy carbon grids (R1.2/1.3, 400 mesh copper, Electron Microscopy Sciences) or Quantifoil holy carbon grids (R1.2/1.3, 400 mesh gold, Quantifoil Micro Tools). The grids were blotted for $7.5 \mathrm{~s}$ with a blot force of 16 and $100 \%$ humidity before being plunged into liquid ethane cooled by liquid nitrogen.

\section{Cryo-EM data collection}

Images of NT-NTR1-G $\alpha_{\mathrm{i} 1} \beta_{1} \gamma_{1}-\mathrm{cND}$ were acquired on Titan Krios I at the Harvard Cryo-EM Center for Structural Biology equipped with a BioQuantum K3 Imaging Filter (slit width $20 \mathrm{eV}$ ) and a $\mathrm{K} 3$ direct electron detector (Gatan) and operating at an acceleration voltage of $300 \mathrm{kV}$. Images were recorded at a defocus range of $-1.2 \mu \mathrm{m}$ to $-2.5 \mu \mathrm{m}$ with a nominal magnification of $105,000 \times$, resulting in a pixel size of $0.825 \AA$. Each image was dose-fractionated into 38 movie frames with a total exposure time of $1.5 \mathrm{~s}$, resulting in a total dose of $\sim 57$ electrons per $\AA^{2}$. SerialEM was used for data collection ${ }^{47}$.

\section{Image processing}


A total of 23,677 movie stacks, which were collected during two sessions, were motion corrected and electron-dose weighted using MotionCor2 (ref. ${ }^{48}$ ). Parameters of the contrast transfer function were estimated from the motion-corrected micrographs using CTFFIND4 $\left(\right.$ ref $^{49}$ ). To generate a reference, particles from 10 micrographs were picked manually in EMAN2.2 (ref. ${ }^{50}$ ), crYOLO $^{51}$ was then trained for picking particles automatically. All subsequent $2 \mathrm{D}$ and $3 \mathrm{D}$ analyses were performed using RELION-3.0 or RELION-3.1-beta ${ }^{52}$.

$1,726,457$ particles were selected after several rounds of $2 \mathrm{D}$ classification from 4,367,542 autopicked particles. Density map of the human NTR1 in complex with the agonist JMV449 and the heterotrimeric $\mathrm{G}_{\mathrm{i} 1}$ protein $(\mathrm{EMDB}-20180)^{8}$ was low-pass filtered to $20 \AA$ and used as the initial model for the first round of $3 \mathrm{D}$ classification, yielding five different classes. Two classes of the NT-NTR1-G $\alpha_{i 1} \beta_{1} \gamma_{1}-\mathrm{cND}$ complex were relatively better resolved and particles from these two classes were subject to 3D refinements. Bayesian polishing was then performed, followed by 3D refinement and post-processing, yielding two density maps at resolutions of $4.3 \AA$ (canonical state) and $4.5 \AA$ (noncanonical state), respectively. To further improve the resolution of the core of the complex, masks excluding the nanodisc and the AHD were applied during the 3D refinement, yielding the $4.1 \AA$ (canonical state) and $4.2 \AA$ (noncanonical state) density maps, respectively. Perparticle CTF refinement was performed but did not lead to an improvement in map resolution or quality.

\section{Model building and refinement}


The crystal structures of NT-NTR1 complex (PDB: 4BUO) $)^{30}$ and G protein heterotrimer G $\alpha_{i 1} \beta_{1} \gamma_{2}$ (PDB: $1 \mathrm{GP} 2)^{29}$ and the cryo-EM structure of GaT $\beta_{1} \gamma_{1}$ (PDB: $\left.6 \mathrm{OY} 9\right)^{9}$ were fitted into the density map of the canonical NT-NTR1-G $\alpha_{i 1} \beta_{1} \gamma_{1}-\mathrm{cND}$ complex using the Fit in Map function of

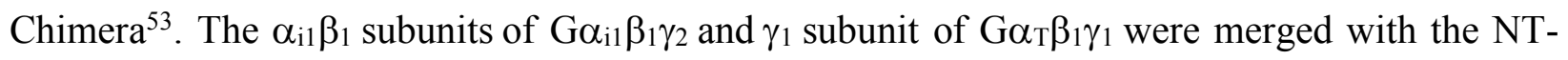
NTR1 structure and the amino acids were modified in Coot version 0.9-pre to match our constructs $^{54}$. The amino acids F291-R299 of NTR1 of the canonical state were mutated to polyalanine due to the lack of sidechain densities. The model was manually adjusted and refined in Coot with torsion, planar peptide, trans peptide and Ramachandran restraints applied. For the noncanonical state, the subunits of the refined atomic model of the canonical state were fitted into the density map as separate rigid bodies. The model was manually adjusted and refined in Coot. For both states, the AHD was extracted from the crystal structure of the human $\mathrm{G}_{\mathrm{i}}$ (PDB: $3 \mathrm{UMR}$ ) and docked into the density as a rigid body using Chimera.

Models were refined with Phenix.real_space_refine ${ }^{55}$. The AHD was not refined due to the lack of sidechain information for this domain. During refinement, the resolution limit was set to match the map resolution determined by the $\mathrm{FSC}=0.143$ criterion in post-processing. Secondary structure, Ramachandran, rotamer, and reference restraints from the JMV449-NTR1-Gi-ScFv16 complex (PDB 6OS9) ${ }^{8}$ were applied throughout refinement. The final models were validated using MolProbity v.4.3.1 (ref. ${ }^{56}$ ) with model statistics provided in Table S1.

\section{Molecular dynamics simulations}


The molecular system for the molecular dynamics (MD) simulations was prepared based on the canonical state structure of NT-NTR1-G $\alpha_{i 1} \beta_{1} \gamma_{1}-\mathrm{cND}$ which was preprocessed with Maestro from Schrödinger ${ }^{57,58}$. Bond orders were assigned, hydrogens added, disulfide bonds created, and het states generated at $\mathrm{pH} 7.0 \pm 2.0$. The sidechains of residues 291 to 299 were assigned and the truncated residues 273 to 290 in NTR1 construct were added with the Crosslink Proteins tool of Maestro $^{57,58}$.

The membrane and solvent environment, as well as the input files for Amber were generated using the Membrane Builder tool of CHARMM-GUI ${ }^{59,60}$. The terminal groups of each chain were patched with standard $\mathrm{N}$-terminus and $\mathrm{C}$-terminus patch residues, except for the $\mathrm{N}$-terminus of $\mathrm{G} \alpha$ for which a GLYP patch residue was used. For orienting the complex appropriately, the PPM (Positioning of Proteins in Membrane) server of the OPM (Orientations of Proteins in Membranes) database was used ${ }^{61}$. A lipid bilayer containing a total of 527 lipids, composed of a 3:2 molar ratio of POPC to POPG, was added to the aligned complex with Membrane Builder ${ }^{59,60}$. A rectangular solvation box was added by adding water layers of at least $22.5 \AA$ above and below the membrane. The system was ionized and neutralized by adding $50 \mathrm{mM}$ of sodium and chloride ions. The resulting system contained a total of 286,109 atoms.

In total, 12 simulations of the prepared system were run using Amber18 (ref. ${ }^{62}$ ). The Amber FF14SB ${ }^{63}$ and Amber Lipid17 (ref. ${ }^{64}$ ) force fields were used for the proteins and the lipid bilayer, respectively. The TIP3P model ${ }^{65}$ was used for the water molecules. During the energy minimization, 2500 steps of steepest descent followed by 2500 steps of conjugate gradient were carried out. The equilibration steps were carried out according to the standard Membrane Builder 
protocols $^{66}$. The production MD simulations were carried out at $310 \mathrm{~K}$ and 1 bar in an NPT ensemble using a Monte Carlo barostat and a Langevin thermostat. The cutoff for the nonbonded interactions was set to $10 \AA$, and the particle mesh Ewald method was used for the long-range electrostatic interactions. Hydrogen mass repartitioning was enabled, and a time step of 4 fs applied. Postprocessing was carried out with AmberTools 18 and VMD 1.9.4 (ref. ${ }^{62}$ ) The simulation lengths of the runs were between $600 \mathrm{~ns}$ and $1 \mu \mathrm{s}$.

\section{References}

43. Egloff, P., Deluigi, M., Heine, P., Balada, S. \& Plückthun, A. A cleavable ligand column for the rapid isolation of large quantities of homogeneous and functional neurotensin receptor 1 variants from E. coli. Protein Expr. Purif. 108, 106-114 (2015).

44. Hillenbrand, M., Schori, C., Schöppe, J. \& Plückthun, A. Comprehensive analysis of heterotrimeric G-protein complex diversity and their interactions with GPCRs in solution. Proc. Natl. Acad. Sci. U. S. A. 112, E1181-90 (2015).

45. Delaglio, F. et al. NMRPipe: A multidimensional spectral processing system based on UNIX pipes. J. Biomol. NMR 6, 277-293 (1995).

46. Ehrenmann, J. et al. High-resolution crystal structure of parathyroid hormone 1 receptor in complex with a peptide agonist. Nat. Struct. Mol. Biol. 25, 1086-1092 (2018).

47. Schorb, M., Haberbosch, I., Hagen, W. J. H., Schwab, Y. \& Mastronarde, D. N. Software tools for automated transmission electron microscopy. Nat. Methods 16, 471-477 (2019).

48. Zheng, S. Q. et al. MotionCor2: Anisotropic correction of beam-induced motion for improved cryo-electron microscopy. Nature Methods 14, 331-332 (2017). 
49. Rohou, A. \& Grigorieff, N. CTFFIND4: Fast and accurate defocus estimation from electron micrographs. J. Struct. Biol. 192, 216-221 (2015).

50. Tang, G. et al. EMAN2: An extensible image processing suite for electron microscopy. $J$. Struct. Biol. 157, 38-46 (2007).

51. Wagner, T. et al. SPHIRE-crYOLO is a fast and accurate fully automated particle picker for cryo-EM. Commun. Biol. 2, (2019).

52. Zivanov, J. et al. New tools for automated high-resolution cryo-EM structure determination in RELION-3. Elife 7, (2018).

53. Pettersen, E. F. et al. UCSF Chimera - A visualization system for exploratory research and analysis. J. Comput. Chem. 25, 1605-1612 (2004).

54. Emsley, P. \& Cowtan, K. Coot: Model-building tools for molecular graphics. Acta Crystallogr. Sect. D Biol. Crystallogr. 60, 2126-2132 (2004).

55. Afonine, P. V. et al. Real-space refinement in PHENIX for cryo-EM and crystallography. Acta Crystallogr. Sect. D Struct. Biol. 74, 531-544 (2018).

56. Williams, C. J. et al. MolProbity: More and better reference data for improved all-atom structure validation. Protein Sci. 27, 293-315 (2018).

57. Epik, S. Schrödinger Suite 2018-2 Protein Preparation Wizard. (2019).

58. Madhavi Sastry, G., Adzhigirey, M., Day, T., Annabhimoju, R. \& Sherman, W. Protein and ligand preparation: Parameters, protocols, and influence on virtual screening enrichments. J. Comput. Aided. Mol. Des. 27, 221-234 (2013).

59. Jo, S., Kim, T., Iyer, V. G. \& Im, W. CHARMM-GUI: A web-based graphical user 
interface for CHARMM. J. Comput. Chem. 29, 1859-1865 (2008).

60. Lee, J. et al. CHARMM-GUI Membrane Builder for Complex Biological Membrane Simulations with Glycolipids and Lipoglycans. J. Chem. Theory Comput. 15, 775-786 (2019).

61. Lomize, M. A., Pogozheva, I. D., Joo, H., Mosberg, H. I. \& Lomize, A. L. OPM database and PPM web server: Resources for positioning of proteins in membranes. Nucleic Acids Res. 40, (2012).

62. Case, D. A. et al. AMBER 2018. (2018).

63. Maier, J. A. et al. ff14SB: Improving the Accuracy of Protein Side Chain and Backbone Parameters from ff99SB. J. Chem. Theory Comput. 11, 3696-3713 (2015).

64. Dickson, C. J. et al. Lipid14: The amber lipid force field. J. Chem. Theory Comput. 10, 865-879 (2014).

65. Jorgensen, W. L., Chandrasekhar, J., Madura, J. D., Impey, R. W. \& Klein, M. L. Comparison of simple potential functions for simulating liquid water. J. Chem. Phys. 79, 926-935 (1983).

66. Lee, J. et al. CHARMM-GUI Input Generator for NAMD, GROMACS, AMBER, OpenMM, and CHARMM/OpenMM Simulations Using the CHARMM36 Additive Force Field. J. Chem. Theory Comput. 12, 405-413 (2016).

Acknowledgments: Cryo-EM data were collected at the Harvard Cryo-Electron Microscopy Center for Structural Biology. We thank F. Koh, P. Egloff, P. Heine, M. Hillenbrand, and J. 
Schöppe for their contribution to the early stages of this project, S. Sterling, R. Walsh, and Z. Li for microscopy support, SBGrid for computing support, M. Deluigi for supervising the signaling experiments, and R. Walker, K. Bayer, P. Imhof and M. Bagherpoor for their advice and discussions regarding the molecular dynamics simulations. M.G. is supported by a Merck-BCMP fellowship. A.B. is supported by the International Retinal Research Foundation, the E. Matilda Ziegler Foundation for the Blind, the Richard and Susan Smith Family Foundation, and the Pew Charitable Trusts. We acknowledge support by NIH grants GM129026 and AI037581 to G.W. and GM131401 to M.L.N. G.W. and A.P. are supported by HFSP RGP0060/2016. A.P. is supported by Swiss National Science Foundation grant 31003A_182334.

Author contributions: M.Z. developed the protocol for making NT-NTR1-G $\alpha_{i 1} \beta_{1} \gamma_{1}-\mathrm{cND}$ complexes, prepared samples, collected negative-stain EM images, and performed biophysical experiments. M.G. prepared cryo-EM grids, obtained and processed the data, and built and refined the atomic models. M.Z. and Z.W. performed binding experiments. C.G. performed MD simulations. J.Y. expressed G $\alpha_{i 1} \beta_{1} \gamma_{1}$. H.W. obtained and processed cryo-EM data. M.Z. and J.S. performed NMR experiments. C. K., L. Me and L. Mo made constructs and performed signaling experiments. A.P. designed and supervised the signaling experiments. F.H. and G.W. initiated the project. A.B., M.L.N., and G.W. designed and supervised the project. M.Z. wrote the paper. M.Z., M.G., Z.W., C.G., J.Y., C. K., A.P., A.B., M.L.N., and G.W. edited the paper.

Competing interests: M.L.N. and G.W. founded the company NOW Scientific to sell assembled cNDs, but a plasmid for expressing the NW9 membrane scaffolding protein is available through 
the Addgene plasmid depository (catalog number 133442) for academic/nonprofit institutions. Otherwise, the authors declare no competing interests.

Data and materials availability: Structural data have been deposited into the Worldwide Protein Data Bank (wwPDB) and the Electron Microscopy Data Bank (EMDB). The EM density maps for the canonical and noncanonical states of NT-NTR1-G $\mathrm{G}_{\mathrm{i}}$ complex in lipid nanodiscs have been deposited under accession codes EMD-XXXX and EMD-XXXX. The masked EM density maps excluding the nanodisc and AHD of the canonical and noncanonical states have been deposited under accession codes EMD-XXXX and EMD-XXXX. The corresponding atomic models have been deposited under accession codes YYYY and YYYY. Other data are available upon reasonable request. 

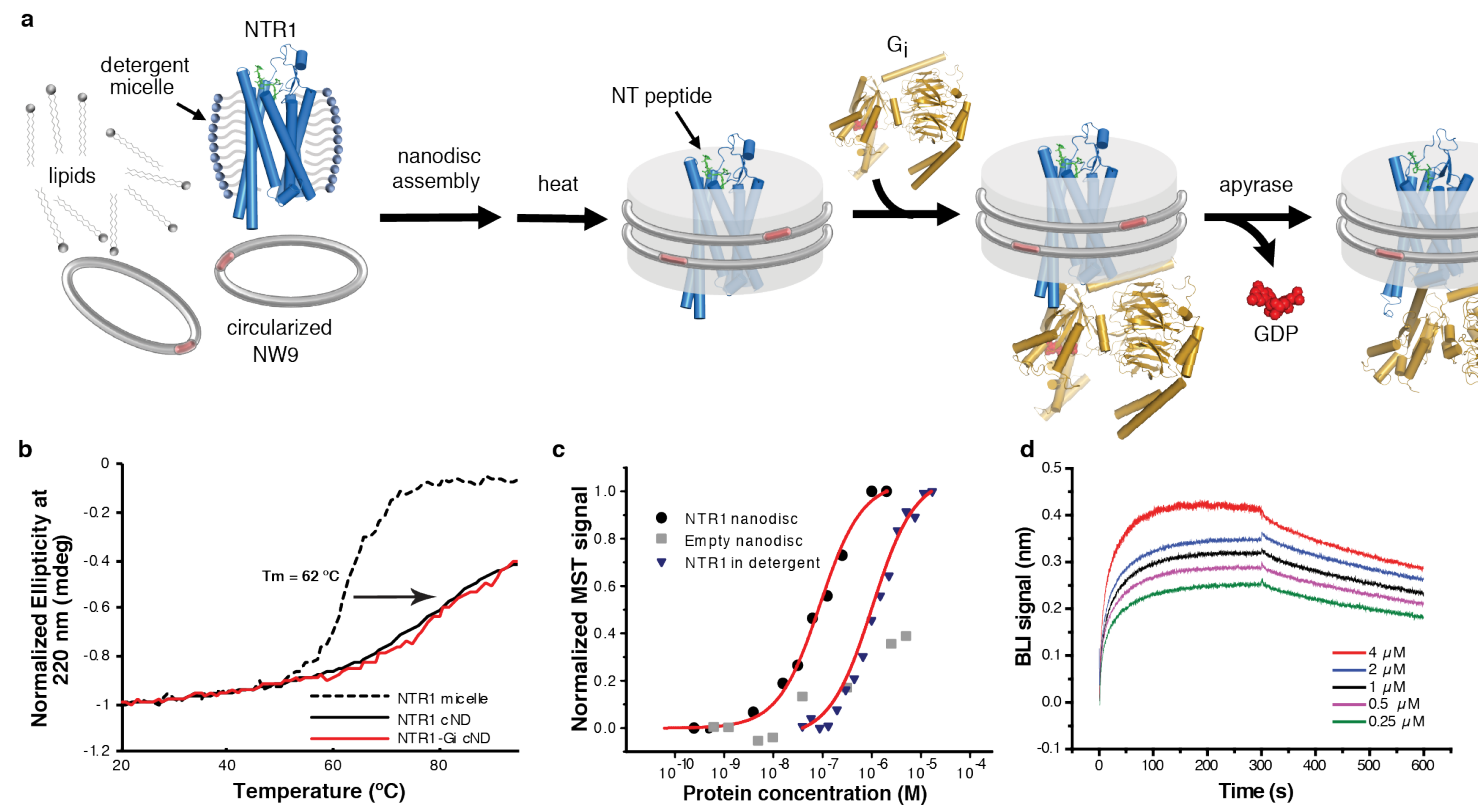

Fig. 1 Assembly and biophysical characterization of the NT-NTR1-G $\alpha_{i 1} \beta_{1} \gamma_{1}$ complex in circularized nanodises (cNDs). a, Schematic showing the assembly of the NT-NTR1-G $\mathrm{G}_{\mathrm{i}}$ complex in lipid nanodiscs using the circularized membrane scaffold protein cNW9. b. Circular dichroism thermostability assays on NT-NTR1 in detergent micelles (dashed black line) with NT-NTR1 (solid black line) and NT-NTR1-G $\mathrm{i}_{\mathrm{i}}$ complex (solid red line) in cNDs. c, Microscale thermophoresis data fitting for the interaction between NT-NTR1 and $\mathrm{G}_{\mathrm{i}}$ in diheptanoylphosphatidylcholine detergent $\left(\mathrm{DH}_{7} \mathrm{PC}\right)$ yields a $\mathrm{K}_{\mathrm{D}}$ of $1400 \pm 100 \mathrm{nM}$ (blue triangles). The interaction between NT-NTR1-cND and $G_{i}$ (black circles) yields a $K_{D}$ of $76 \pm 18 \mathrm{nM}$. Weak binding between empty nanodiscs and $\mathrm{G}_{\mathrm{i}}$ is shown as gray squares. d, Bio-layer interferometry (BLI) traces of $\mathrm{G}_{\mathrm{i}}$ binding to NT-NTR1-cND at five different concentrations. Data fitting results are shown in Extended Data Fig. 4a, b. 


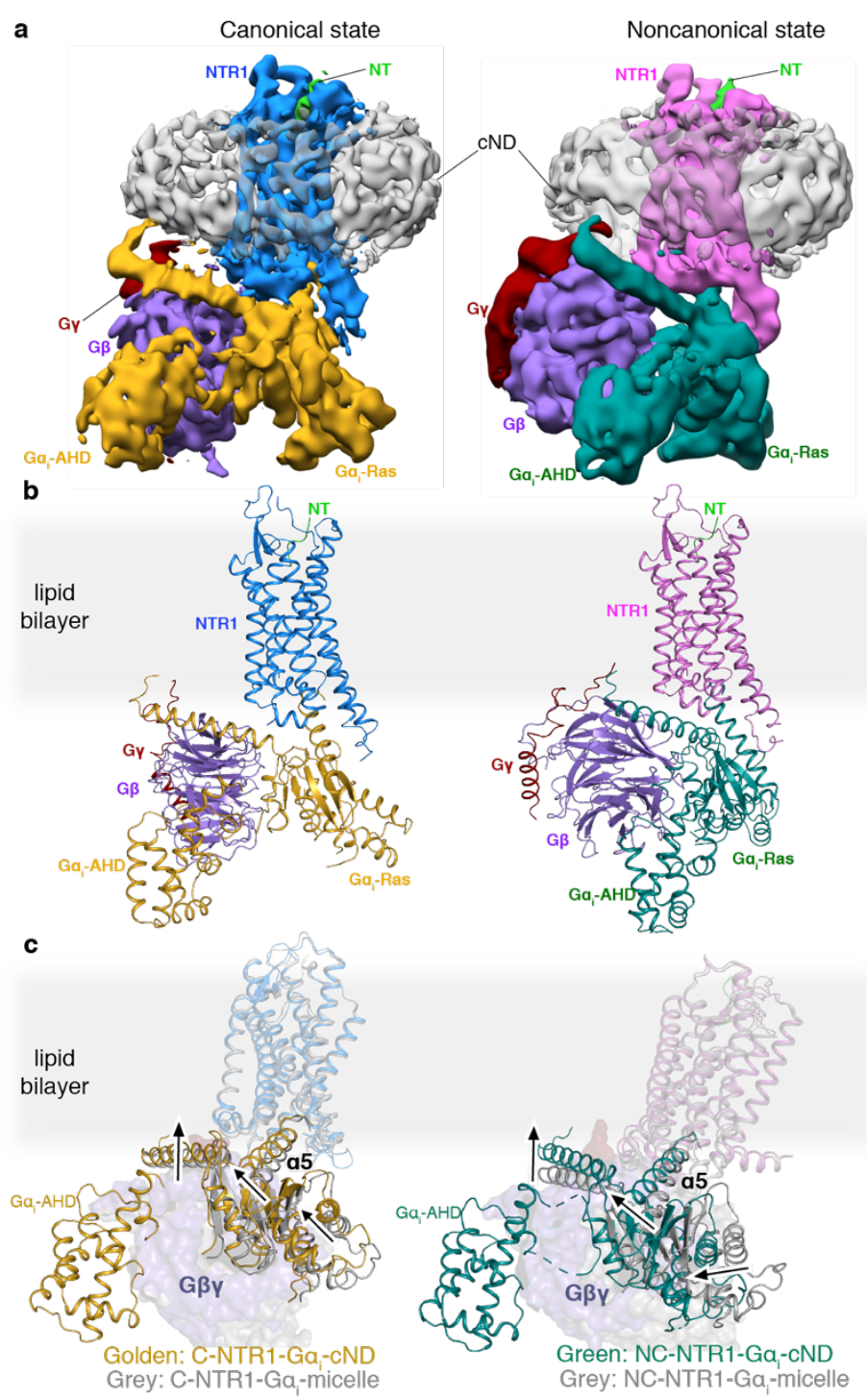

Fig. 2 | Cryo-EM structures of NT-NTR1-Gi-cND. a, Cryo-EM density maps of NT-NTR1-G ${ }^{-}$ cND complex in the canonical state (left) and in the noncanonical state (right). The maps are lowpass filtered to $5 \AA$ and colored by subunit. Higher-resolution maps were obtained by masking out density for the nanodisc and Ga-AHD domain. b, Atomic models of NT-NTR1-G $\mathrm{i}_{\mathrm{i}} \mathrm{cND}$ complex in the canonical state (left) and in the noncanonical state (right). The models are shown in the same orientation as the maps in (a). c, Structural superimposition of C-NT-NTR1-Gi-cND with C- 
bioRxiv preprint doi: https://doi.org/10.1101/2020.06.11.145912; this version posted June 12, 2020. The copyright holder for this preprint (which was not certified by peer review) is the author/funder. All rights reserved. No reuse allowed without permission.

NTR1-scFv16-micelle (left) and NC-NT-NTR1-Gi-cND with NC-NTR1-scFv16-micelle (right).

Structural displacement is highlighted with arrows. The models are superimposed on the NTR1. 


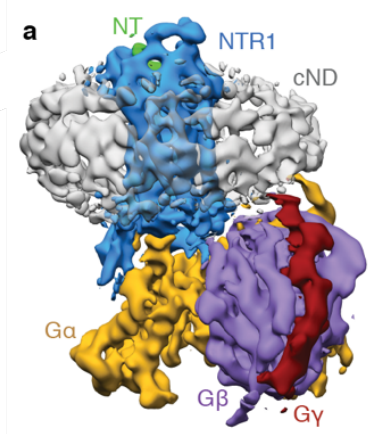

Canonical state

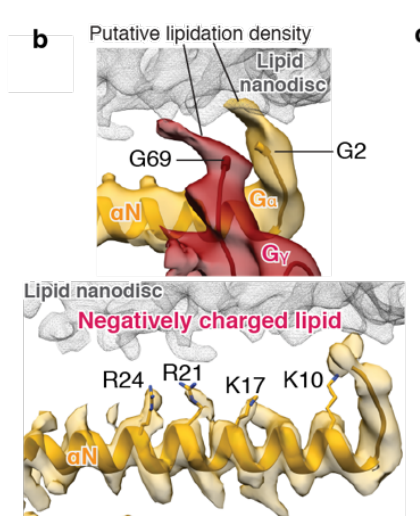

e

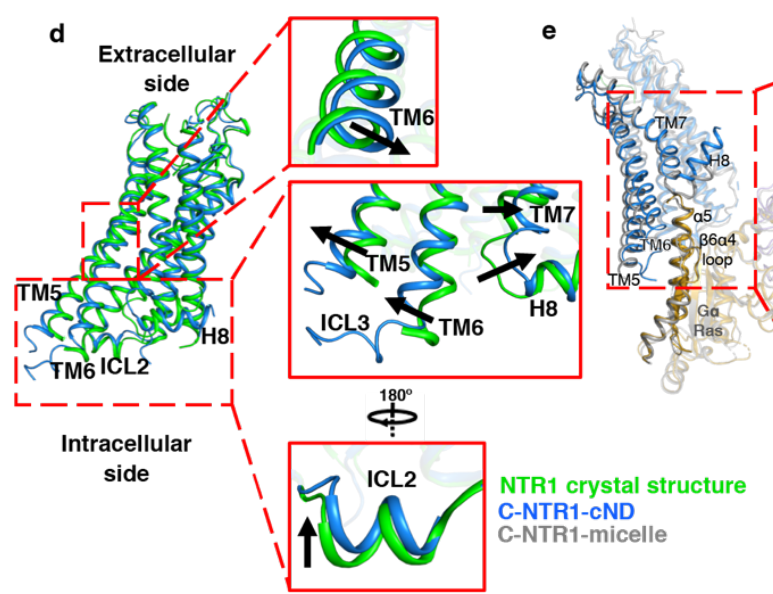

c

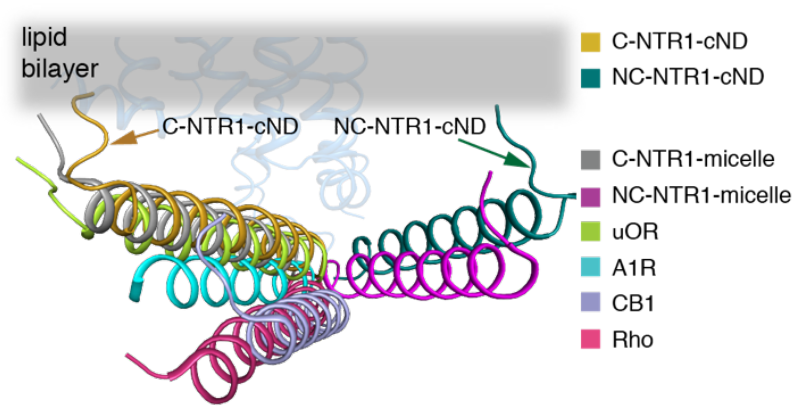

Fig. 3 | Impact of lipid bilayer on the NTR1-Gi complex. a, Cryo-EM density map of the NT-

NTR1-Gi-cND complex in the canonical state. The map is low-pass filtered to $5 \AA$ and colored by subunit. b, Top panel, density for the putative lipid modifications of glycine 2 (G2) of Ga $\alpha_{i 1}$ and glycine 69 (G69) of $\mathrm{G} \gamma_{1}$. Nanodisc density is shown as gray mesh. The density map of the canonical state is low-pass filtered to $5 \AA$. Bottom panel, positively charged residues of the $\alpha \mathrm{N}$ helix of $\mathrm{G \alpha}_{\mathrm{i} 1}$ face the negatively charged lipid bilayer. The $4.1 \AA$ density map of the canonical state is shown. c, Comparison of the $\alpha \mathrm{N}$ helices of GPCR-G $\mathrm{G}_{\mathrm{i}}$ complexes. C-NTR1-cND and NCNTR1-cND indicate the canonical and noncanonical states of the NT-NTR1-Gi complex in lipid nanodiscs. C-NTR1-micelle and NC-NTR1-micelle indicate the canonical and noncanonical states of JMV449-NTR1-G ${ }_{i}$ complex in detergent micelles. Other Class A GPCR-G $\mathrm{G}_{\mathrm{i}}$ complexes: $\mu \mathrm{OR}-$ 
$\mathrm{G}_{\mathrm{i}}$ (lime green; PDB 6DDE), A $\mathrm{A}_{1}-\mathrm{G}_{\mathrm{i}}$ (cyan; PDB 6D9H), CB1-G $($ purple; PDB 6N4B), and Rho$\mathrm{G}_{\mathrm{i}}$ (hot pink; PDB 6CMO). The models are superposed on the GPCR. d, Structural comparison between NTR1 from the canonical state NT-NTR1-G $\mathrm{G}_{\mathrm{i}}$ complex in lipid nanodiscs (blue) and the crystal structure of NTR1 in detergent (green). Zoomed-in views are shown on the right. e, Structural comparison between the canonical states of NTR1-G in lipid bilayer (blue) and detergent (gray), superposed on the Ras-like domain of Ga (gold). Zoomed-in view of the cytoplasmic side of TM5-TM6, ICL3, TM7-H8, as well as the $\alpha 5$ helix and $\beta 6 \alpha 4$ loop of G $\alpha$ is shown on the right. f, Comparison of the location of TM6 relative to the $\alpha 5$ helix of G $\alpha$ in the canonical state NTR1 (blue) in complex with $\mathrm{G}_{\mathrm{i}}$ (gold) with other class A GPCR-G $\mathrm{G}_{\mathrm{i}}$ complex structures, including the canonical state of NTR1-G $\mathrm{G}_{\mathrm{i}}$ in detergent micelle (gray), $\mu \mathrm{OR}-\mathrm{G}_{\mathrm{i}}$ (lime green), Rho- $\mathrm{G}_{\mathrm{i}}$ (hot pink), $\mathrm{A}_{1} \mathrm{R}-\mathrm{G}_{\mathrm{i}}$ (cyan), and CB1-G $\mathrm{G}_{\mathrm{i}}$ (purple). The models are superposed on the Ras-like domain of G $\alpha$. 


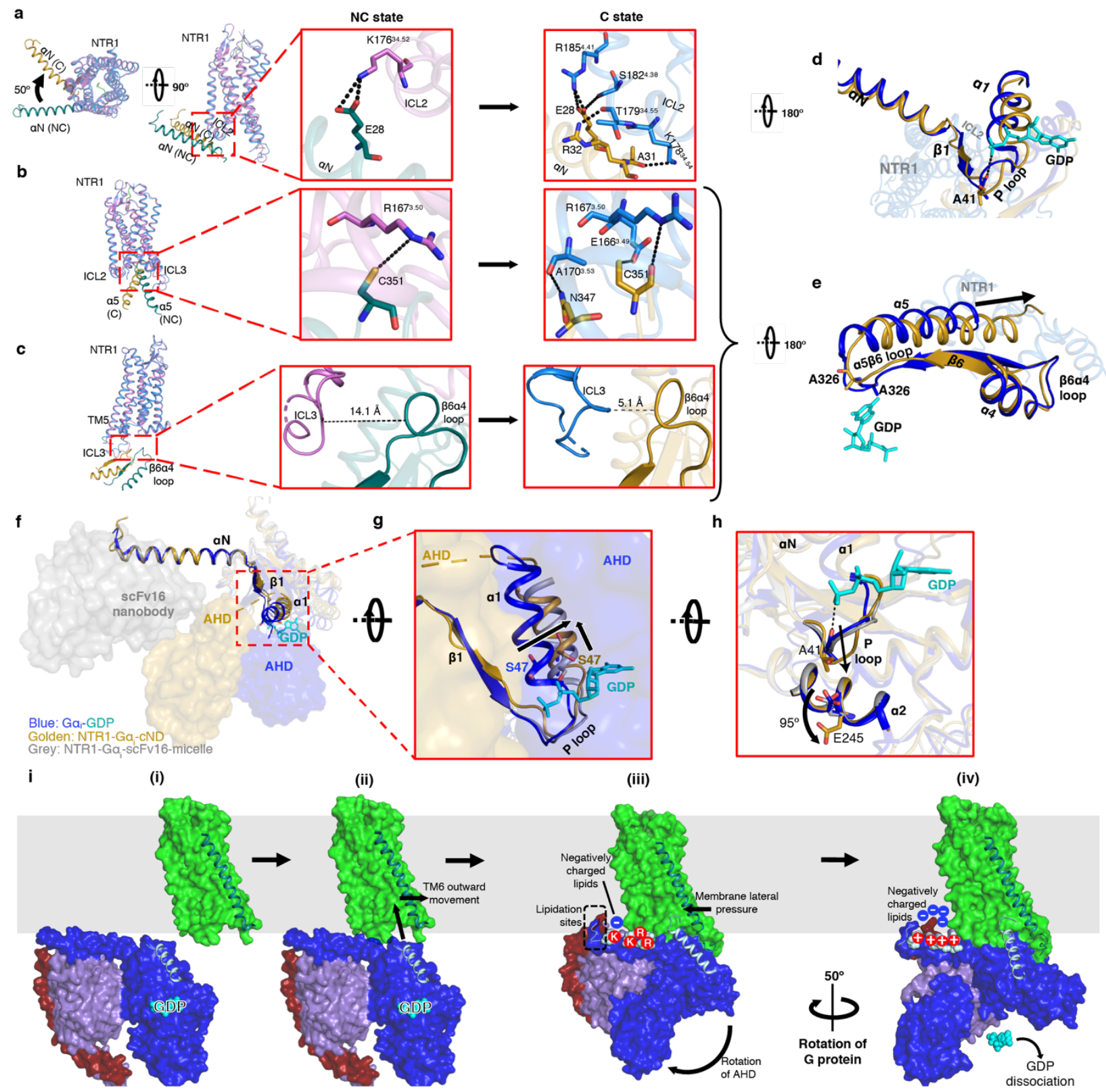

Fig. 4 | Allosteric modulation of the GDP binding pocket by the NTR1-Gi interaction. a-c, Superposition between C-state NTR1 (blue) and $\alpha \mathrm{N}$ helix of G $\alpha$ (gold) with the NC-state NTR1

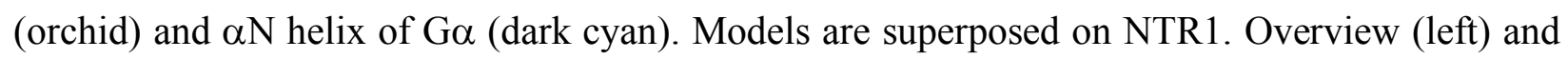
zoomed-in views of the $\mathrm{NC}$ state (middle) and $\mathrm{C}$ state (right) are shown. a, ICL2- $\alpha \mathrm{N}$ helix interactions. Compared to the NC state, the $\alpha \mathrm{N}$ helix of $\mathrm{G} \alpha$ of the $\mathrm{C}$ state is rotated by $50^{\circ}$. b, 
NTR1- $\alpha 5$ helix interactions. c, ICL3- $\beta 6 \alpha 4$ loop interactions. The backbones of ICL3 and $\beta 6 \alpha 4$ are closer in the $\mathrm{C}$ state and form interactions predicted by molecular dynamics simulations (Extended Data Fig. 11a). d, Intracellular view showing perturbation of the P loop in the C state (gold) relative to the crystal structure of GDP-bound $\mathrm{G}_{i}$ (blue; GDP in cyan). e, Intracellular view showing perturbation of the $\alpha 5 \beta 6$ loop in the $\mathrm{C}$ state (gold) relative to the crystal structure of GDPbound $\mathrm{G}_{\mathrm{i}}$ (blue; GDP in cyan). In d-e, the models are superposed on the Ga Ras-like domain. $\mathbf{f}$,

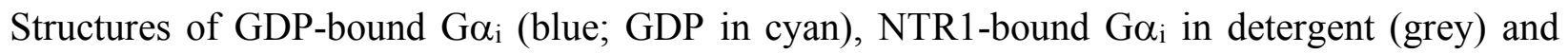
NTR1-bound $G \alpha_{i}$ in lipid bilayer (gold) showing the different locations of the AHD and the stabilizing antibody scFv16. The structures are superposed on $\alpha \mathrm{N}-\beta 1$. g, Zoom-in view showing lateral displacement of $\alpha 1$ helix including S47 from the phosphates of GDP in NTR1-Gi-cND. h, Rotation of E245 in NT-NTR1-G $-\mathrm{G}$ - $N D$ (gold) by $95^{\circ}$ compared to the GDP-G $\mathrm{G}_{\mathrm{i}}$ structure (blue) to accommodate the P-loop. This structural change is not observed in detergent (grey). i, Model of the proposed insertion-rotation mechanism: (i) Lateral diffusion of NTR1 and $\mathrm{G}_{\mathrm{i}}$ in the membrane; (ii) Recognition of NTR1 by $\mathrm{G}_{\mathrm{i}}$, allowing insertion of $\alpha 5$ into the open cavity of NTR1; (iii) Formation of the NC state including displacement of the AHD; (iv) Formation of the C state following rotation of $\mathrm{G}_{\mathrm{i}}$. 


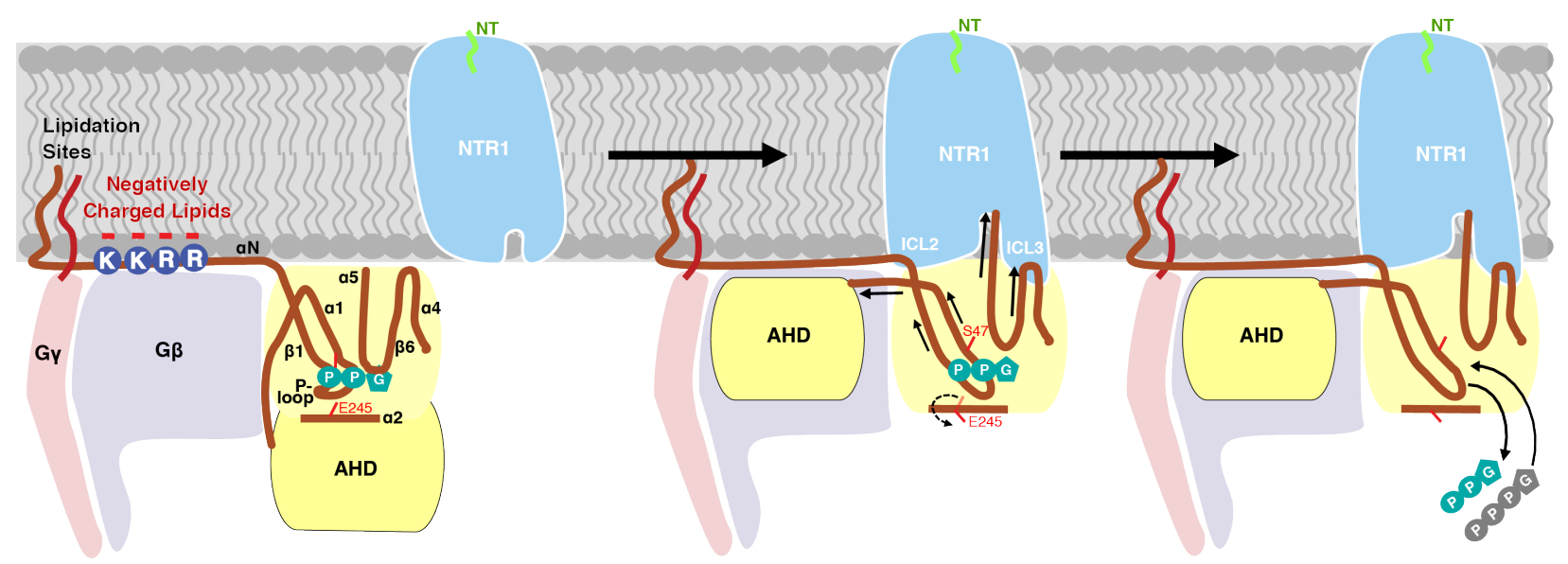

Fig. 5 | Proposed GDP release mechanism. The interaction between $G_{i}$ and NTR1 leads to allosteric modulation of the GDP-binding site via three pathways: (1) The movement of the AHD to $\mathrm{G} \beta$ perturbs the directly linked $\alpha 1$, resulting in $\alpha 1$ dissociation from the phosphate groups of GDP; (2) The interaction between ICL2 of NTR1 and $\alpha \mathrm{N}-\beta 1$ hinge of G protein delivers perturbation into the $\mathrm{P}$-loop though $\beta 1$, resulting in $\mathrm{P}$-loop dissociation from the phosphate groups of GDP, which is coupled with a $95^{\circ}$ rotation of the sidechain of E245 on $\alpha 2$; (3) The interactions between $\alpha 5$ and core of NTR1 and between $\beta 6 \alpha 4$ loop and ICL3 pull the $\alpha 5 \beta 6$ loop away from the guanine ring of GDP. The multi-point coordination of these structural elements leads to dissociation of both the phosphates and the guanine ring of GDP from $\mathrm{G}_{\mathrm{i}}$, and thus dissociation of the entire GDP. Release of GDP creates a free nucleotide-binding pocket available for subsequent GTP binding, thus completing the GDP/GTP exchange process. 

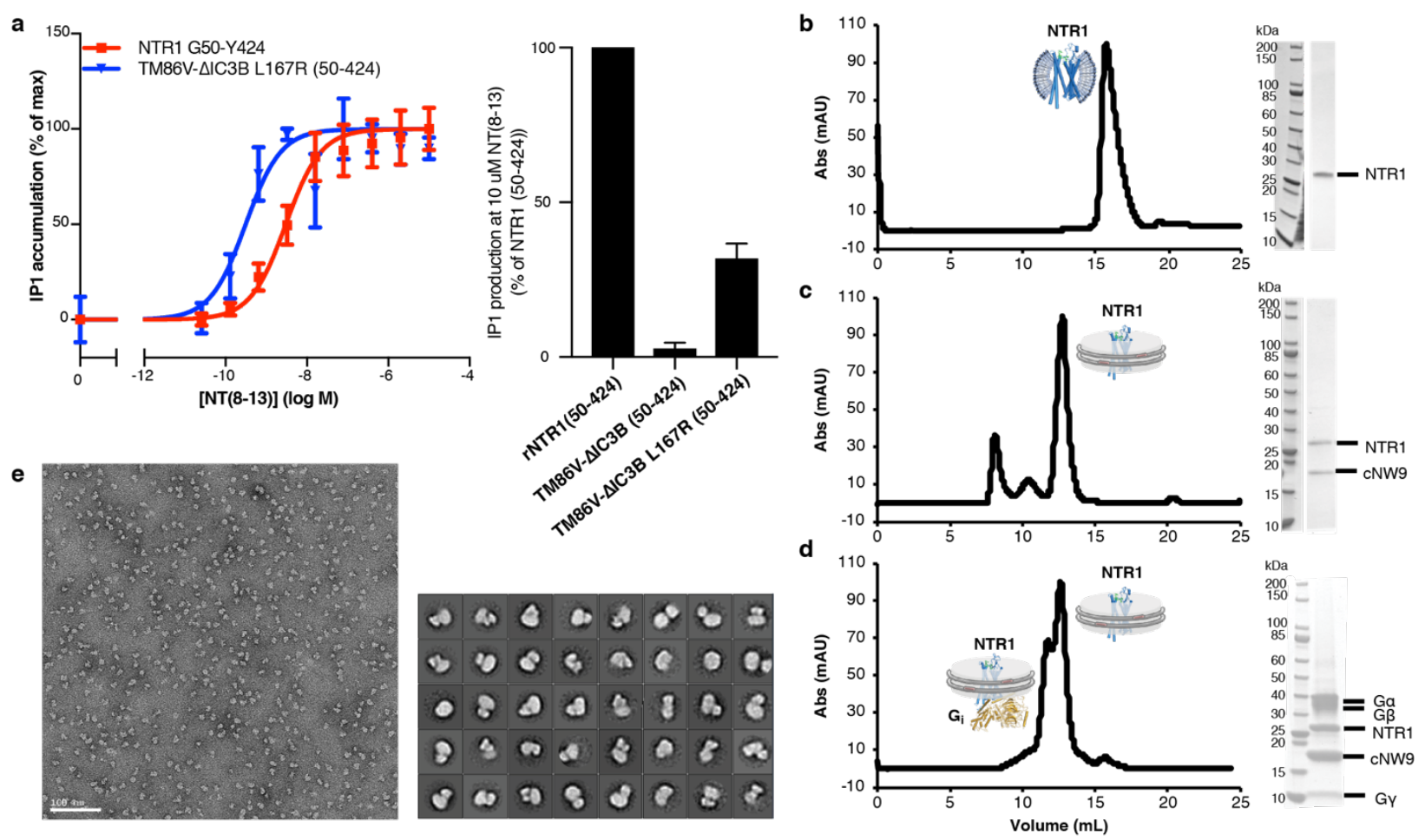

Extended Data Fig. 1 | Signaling competency and preparation of NT-NTR1-G complex in $_{i}$

cNDs. a, Signaling competency of NTR1 constructs. Wild-type NTR1 (50-424) or NTR1 variants were transiently transfected into HEK293T/17 cells, and activation of $\mathrm{G} \alpha_{\mathrm{q}}$ signaling was quantified by measuring of inositol-1-phosphate (IP1) accumulation after stimulation with $\mathrm{NT}_{8-13}$. Data were normalized to receptor expression at the cell surface and represented as mean \pm SEM of 4 independent experiments performed in duplicate. Left, dose dependent IP1 production expressed as percentage of IP1 accumulation at maximal ligand concentration. Fitting of the curves result in EC50 of $2.664 \mathrm{nM}$ for wild-type NTR1 and $0.2188 \mathrm{nM}$ for TM86V $\triangle \mathrm{IC} 3 \mathrm{~B}$ L167R. Right, bar graph showing IP1 production level at $10 \mu \mathrm{M}$ agonist $\mathrm{NT}_{8-13}$. The NTR1 variant TM86V $\Delta \mathrm{IC} 3 \mathrm{~B}$ lacking the L167R back mutation exhibits no IP1 production, suggesting the critical role of R167 3.50 in signal transduction. b-d, Size-exclusion chromatograms and corresponding SDSPAGE gels for NTR1 in $\mathrm{DH}_{7} \mathrm{PC}$ detergent micelles (b), NTR1 in POPC/POPG cNW9 nanodiscs (c) and NTR1-G $\mathrm{G}_{\mathrm{i}}$ complex in POPC/POPG cNW9 nanodiscs (d). e, Fractions corresponding to the 
bioRxiv preprint doi: https://doi.org/10.1101/2020.06.11.145912; this version posted June 12, 2020. The copyright holder for this preprint (which was not certified by peer review) is the author/funder. All rights reserved. No reuse allowed without permission.

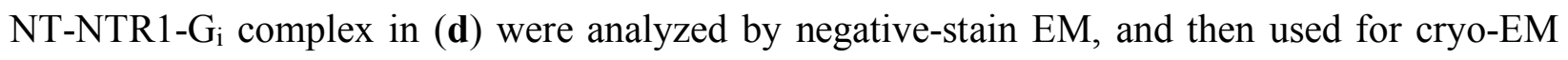
structure determination. Top, representative negative-stain EM micrograph of NT-NTR1-G complexes in cNDs. Bottom, 2D class averages. 
a
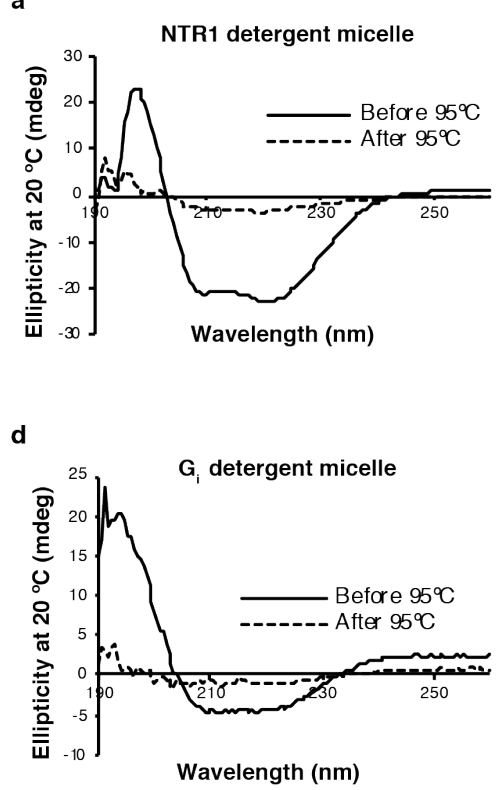

b

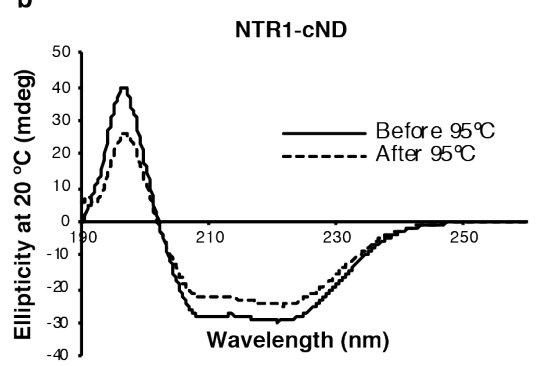

e

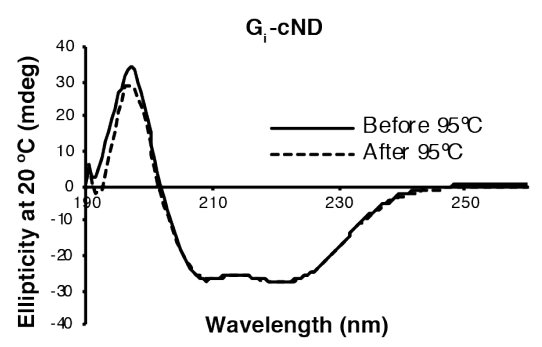

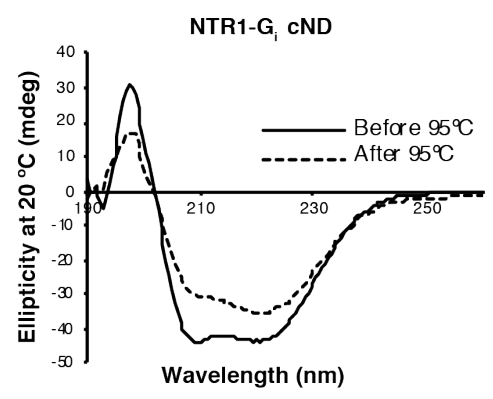

f

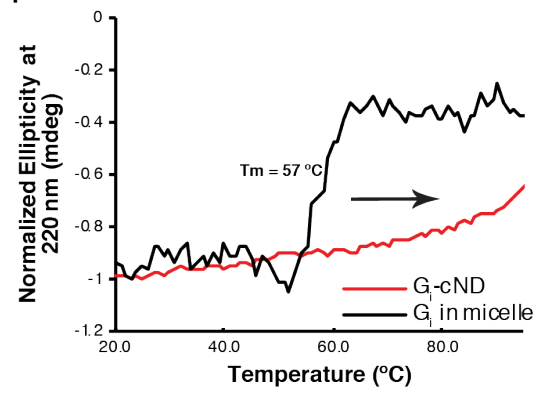

Extended Data Fig. 2 | Thermostability enhancement of NTR1, $G_{i}$, and NTR1-G complexes by incorporation into cNDs. a-e, Circular Dichroism (CD) spectra at $20{ }^{\circ} \mathrm{C}$ before (solid line) and after (dashed line) treatment at $95^{\circ} \mathrm{C}$ of (a) NTR1 in $\mathrm{DH}_{7} \mathrm{PC}$ detergent micelles; (b) NTR1 in cNDs; (c) NTR1-G $\mathrm{G}_{\mathrm{i}}$ complex in cNDs; (d) $\mathrm{G}_{\mathrm{i}}$ in detergent micelle; (e) $\mathrm{G}_{\mathrm{i}}$ in cNDs. $\mathrm{G}_{\mathrm{i}}$ was reconstituted into cNDs by incubation with POPC/POPG lipid, cNW9, and cholate, followed by detergent removal and size-exclusion chromatography. f, Temperature-dependent CD signals of $\mathrm{G}_{\mathrm{i}}$ in detergent micelles (black) and cNDs (red) at $220 \mathrm{~nm}$. The melting temperature (Tm) of cNDs is $93{ }^{\circ} \mathrm{C}$ (data not shown) and therefore does not affect transitions before this temperature. NTR1 and $\mathrm{G}_{\mathrm{i}}$ account for at least $50 \%$ of CD signals even in the presence of cNDs. NTR1 in detergent micelles irreversibly unfolds during temperature increase with a Tm of $62{ }^{\circ} \mathrm{C}$. In contrast, NTR1cND changes structure around $80{ }^{\circ} \mathrm{C}$ and does not lose much secondary structure after decreasing temperature to $20^{\circ} \mathrm{C}$. Similar observations were made for $\mathrm{G}_{\mathrm{i}}$, where the protein irreversibly and completely unfolds with $\mathrm{Tm}$ of $57^{\circ} \mathrm{C}$ in detergent micelles but displays no clear transition temperature in cNDs even until $95^{\circ} \mathrm{C}$. For the NTR1-G 
bioRxiv preprint doi: https://doi.org/10.1101/2020.06.11.145912; this version posted June 12, 2020. The copyright holder for this preprint (which was not certified by peer review) is the author/funder. All rights reserved. No reuse allowed without permission.

observed around $82{ }^{\circ} \mathrm{C}$. These observations indicate that lipid bilayers improve the stability of NTR1, $\mathrm{G}_{\mathrm{i}}$ and NTR1-G $\mathrm{i}$ complexes relative to detergent micelles. 

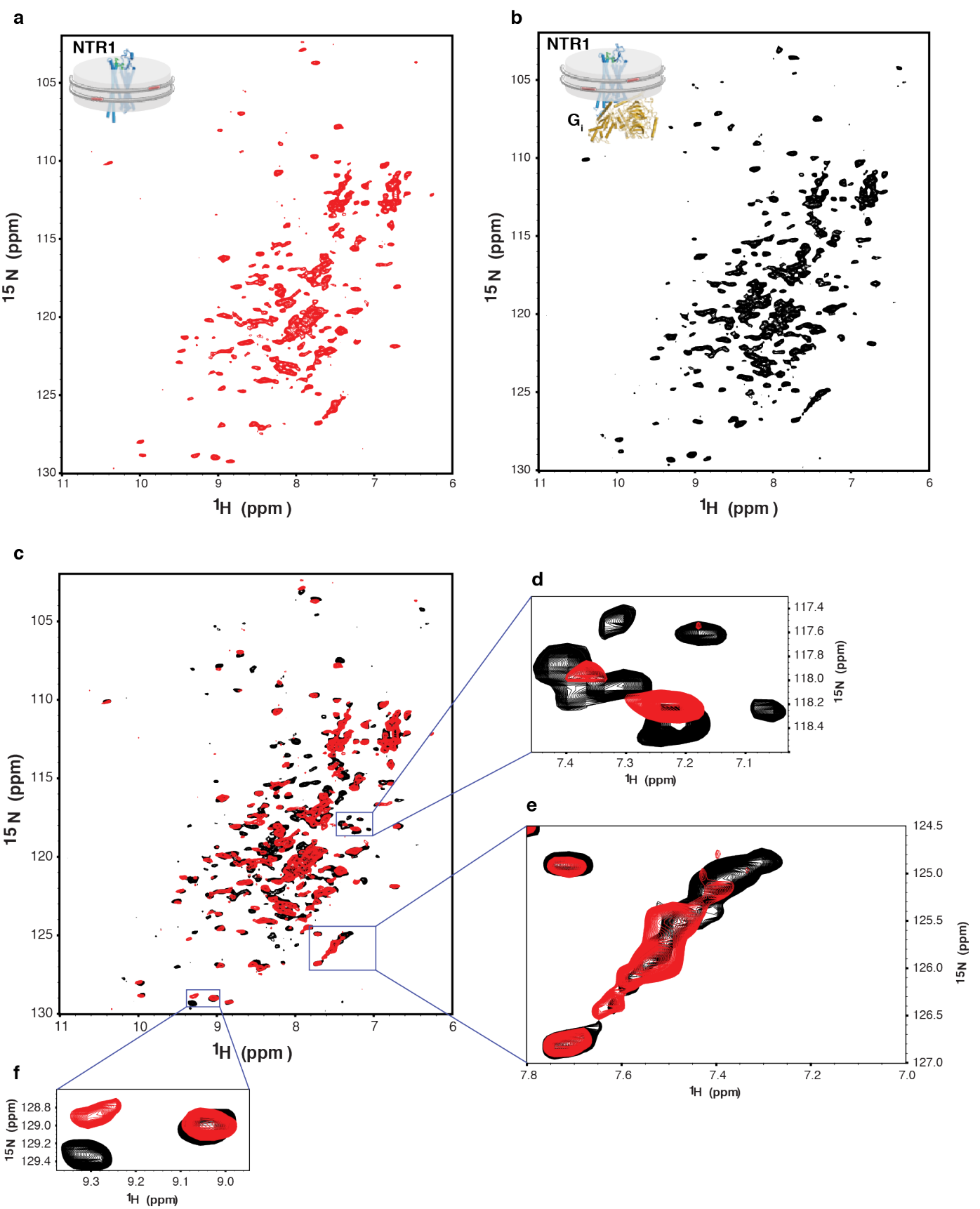

Extended Data Fig. 3 | Characterization of the interaction between NT-NTR1 and $G_{i}$ in cNDs by two-dimensional ${ }^{1} \mathrm{H},{ }^{15} \mathrm{~N}$-TROSY HSQC NMR spectroscopy. a-b, NMR spectrum of ${ }^{15} \mathrm{~N}$ labeled NT-NTR1 in cNDs in the absence (a) and presence (b) of $\mathrm{G}_{\mathrm{i}}$. c, Overlay of (a) (red) onto (b) (black) showing structural and dynamical changes of NT-NTR1 upon binding to $\mathrm{G}_{\mathrm{i}}$ in cNDs. d, A region showing conformational stabilization of NTR1. More peaks could be observed in the 
presence of $G_{i}$, suggesting that NTR1 is highly dynamic in the absence of $G_{i}$ and resonances are averaged out among a wide range of conformers resulting in low signal-to-noise ratio and even disappeared peaks. Upon interaction with $\mathrm{G}_{\mathrm{i}}$, NTR1 is stabilized into fewer conformers and becomes less dynamic, which leads to better signal-to-noise ratio and more resonances being observable. e, A region showing dynamically slow-exchange shift of NTR1 upon interaction with $\mathrm{G}_{\mathrm{i}}$. f, A region showing chemical shift perturbation of NTR1, suggesting conformational change of NTR1 upon binding to $\mathrm{G}_{\mathrm{i}}$ in cNDs. 

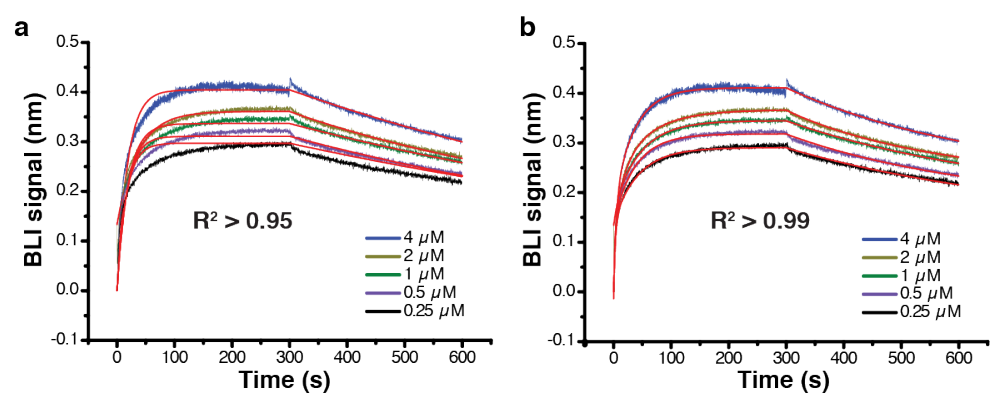

\begin{tabular}{|c|c|c|c|}
\hline & $\mathrm{K}_{\text {on }}\left(1 / \mathrm{M}^{*} \mathrm{~S}\right)$ & $\mathrm{K}_{\text {off }}(1 / \mathrm{s})$ & $\mathrm{K}_{\mathrm{D}}(\mathrm{nM})$ \\
\hline Mode 1 & $2.9 \pm 0.1 \times 10^{5}$ & $1.7 \pm 0.2 \times 10^{-3}$ & $5.8 \pm 1.6$ \\
\hline Mode 2 & $6.4 \pm 0.3 \times 10^{4}$ & $2.5 \pm 0.2 \times 10^{-3}$ & $38 \pm 11$ \\
\hline
\end{tabular}
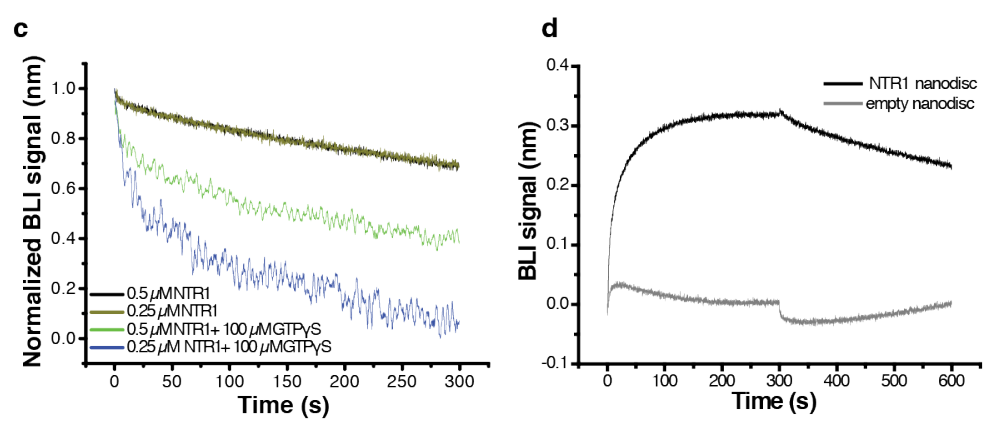

Extended Data Fig. 4 | Characterization of the binding kinetics between NT-NTR1 and $G_{i}$ in cNDs. a-b, Fitting of Bio-Layer Interferometry (BLI) traces of $\mathrm{G}_{\mathrm{i}}$ binding to NT-NTR1-cND using one binding mode (a) and two binding mode (b) shows better fitting using two binding mode. Below, a table showing $\mathrm{k}_{\mathrm{on}}, \mathrm{k}_{\mathrm{off}}$ and $\mathrm{K}_{\mathrm{D}}$ from the two binding mode fitting. $\mathrm{c}$, Dissociation between $\mathrm{G}_{\mathrm{i}}$ and NT-NTR1-cND in the absence (black and brown) and presence (green and blue) of GTP $\gamma \mathrm{S}$, showing faster dissociation of the complex in the presence of GTP $\gamma \mathrm{S}$, suggesting the capability of the NTR1-G $\alpha_{i 1} \beta_{1} \gamma_{1}$ complex in cNDs to perform GDP/GTP exchange. d, Association and dissociation kinetics of $\mathrm{G}_{\mathrm{i}}$ binding to NT-NTR1-cND (dark) and empty cND (gray), showing much slower association and faster dissociation of $\mathrm{G}_{\mathrm{i}}$ binding to empty cND compared to NT-NTR1cND, suggesting that interaction between $\mathrm{G}_{\mathrm{i}}$ and NT-NTR1-cND is driven by $\mathrm{G}_{\mathrm{i}}$ binding to NTR1 rather than to the nanodisc. 
a

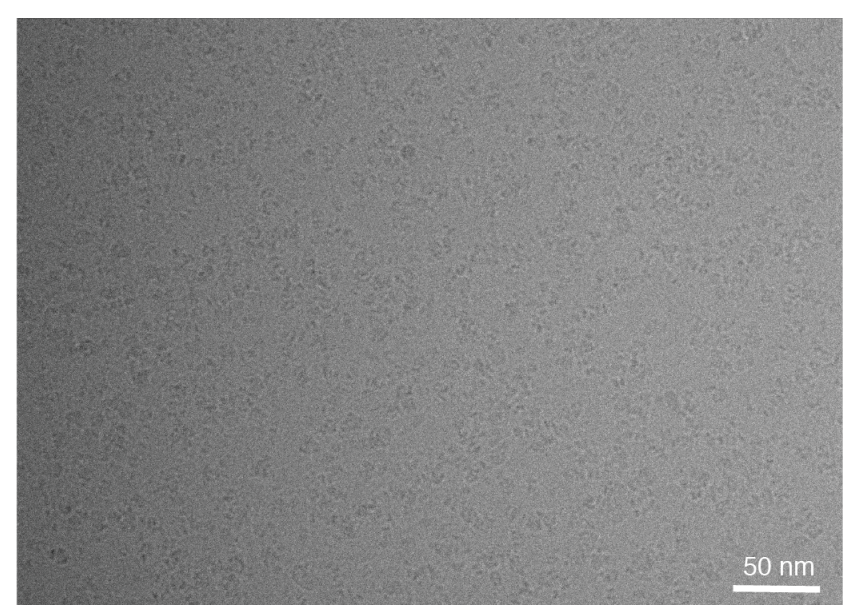

b

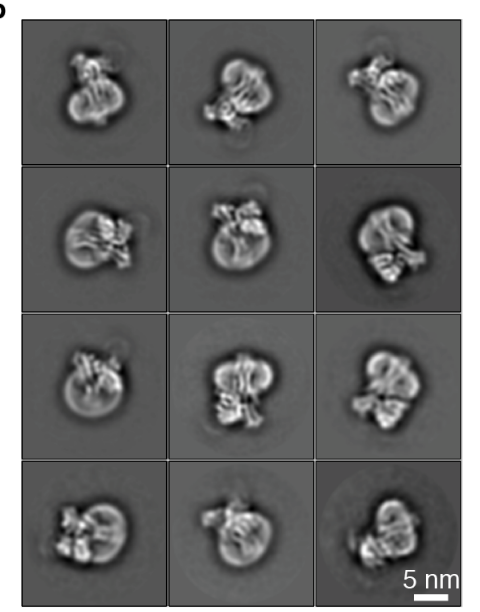

c

23,677 motion-corrected movie stacks
crYOLO autopick

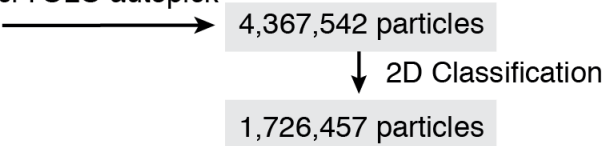

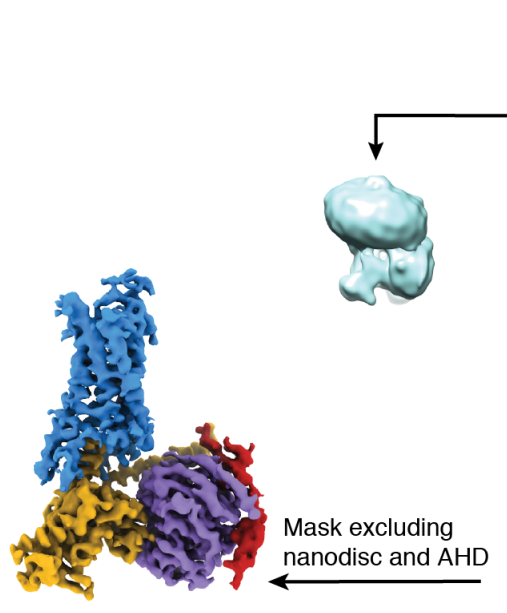

canonical state, $4.1 \AA$
575,791 particles

d

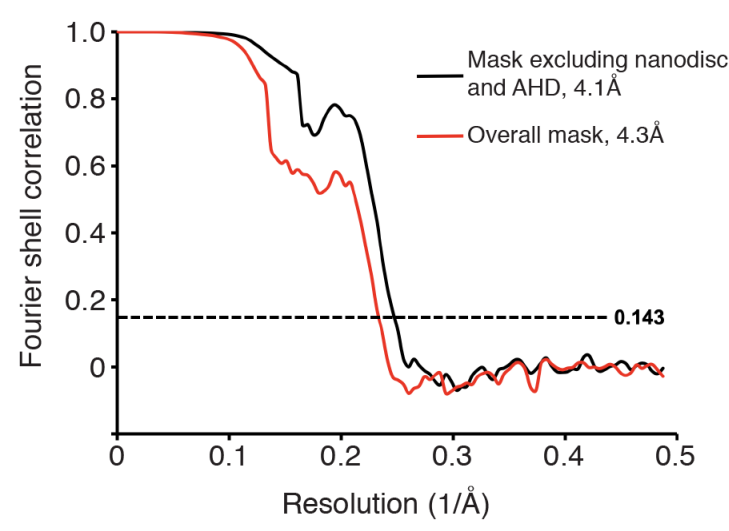

3D Classification

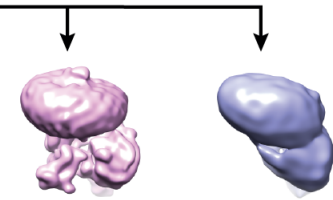

3D Refinement

Bayesian Polishing

3D Refinement

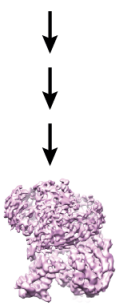

Mask excluding

nanodisc and AHD

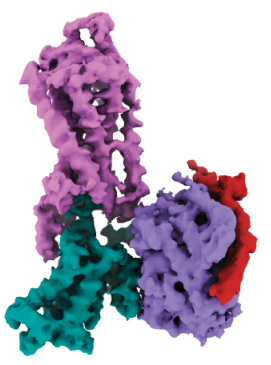

324,002 particles

noncanonical state, $4.2 \AA$

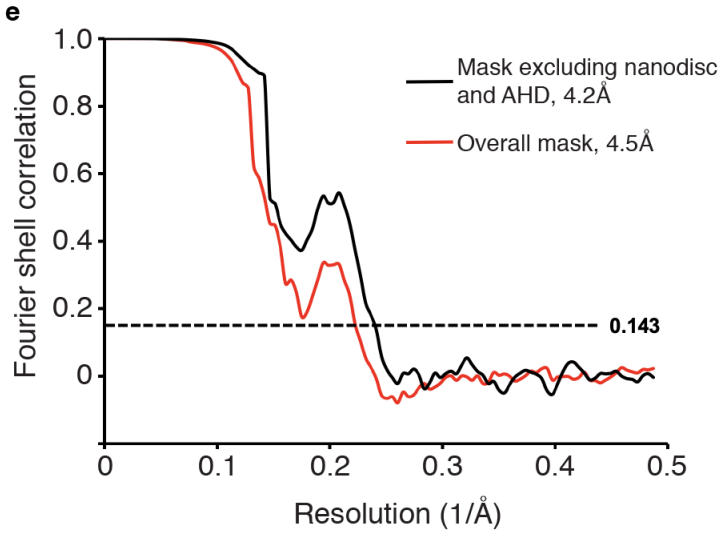

Extended Data Fig. 5 | Cryo-EM data processing. a, Representative micrograph showing the distribution of NT-NTR1-Gi-cND particles in vitreous ice. b, Selected two-dimensional class 
averages showing secondary structure features. The cND has an approximate diameter of $9 \mathrm{~nm}$. c, Simplified flow chart of the cryo-EM processing. Two datasets were collected and processed similarly; the number of particles shown here are a conflation of both datasets. Two well-resolved classes corresponding to canonical and noncanonical states were identified. Further rounds of classification did not improve the resolution or map quality. d-e, Fourier shell correlation (FSC) curves for the canonical state (d) and noncanonical state (e) with masks that either include or exclude the cND and AHD. 

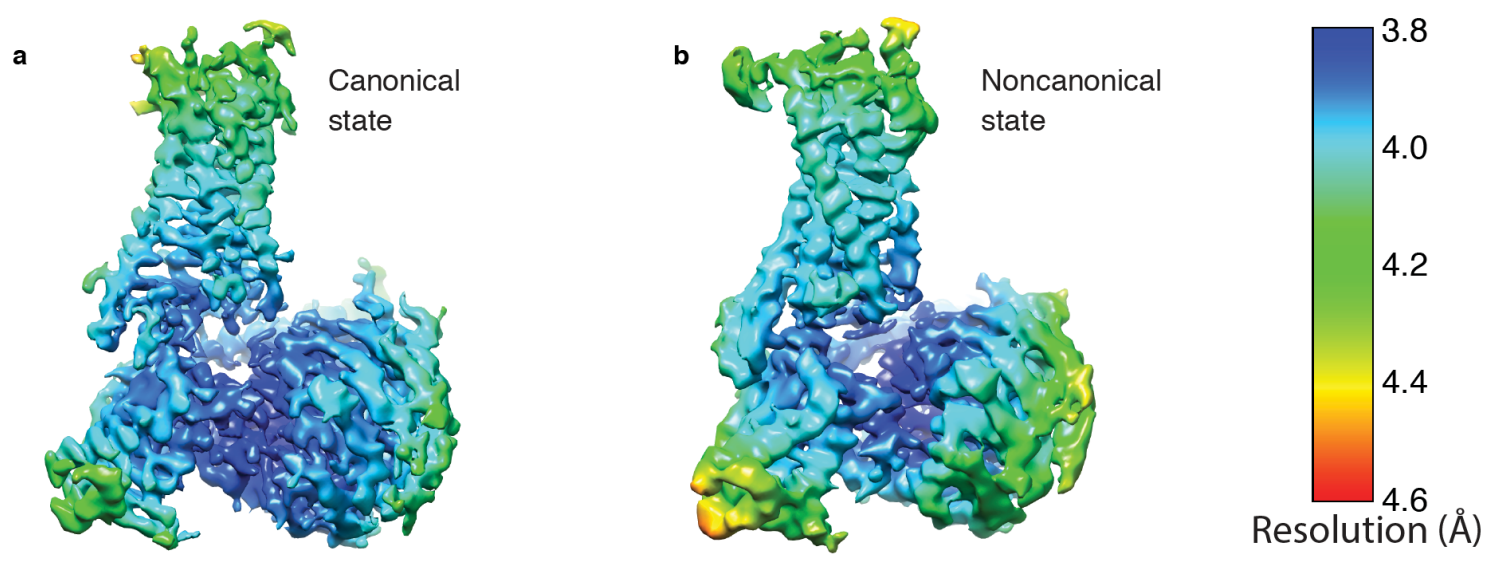

c
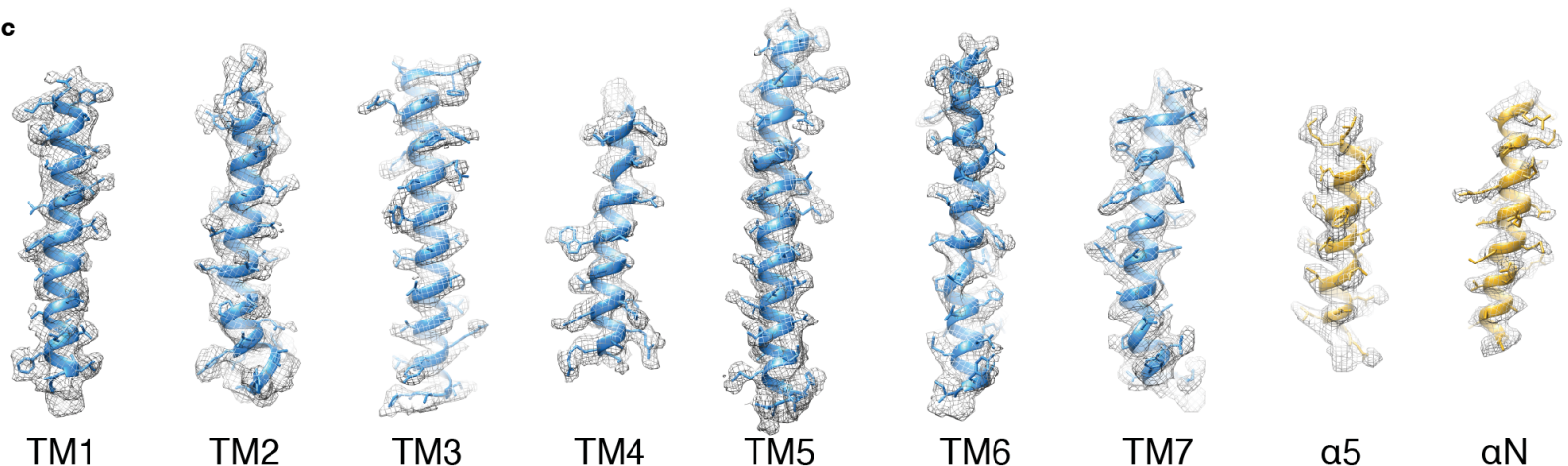

TM1

TM2

TM3

TM4

d
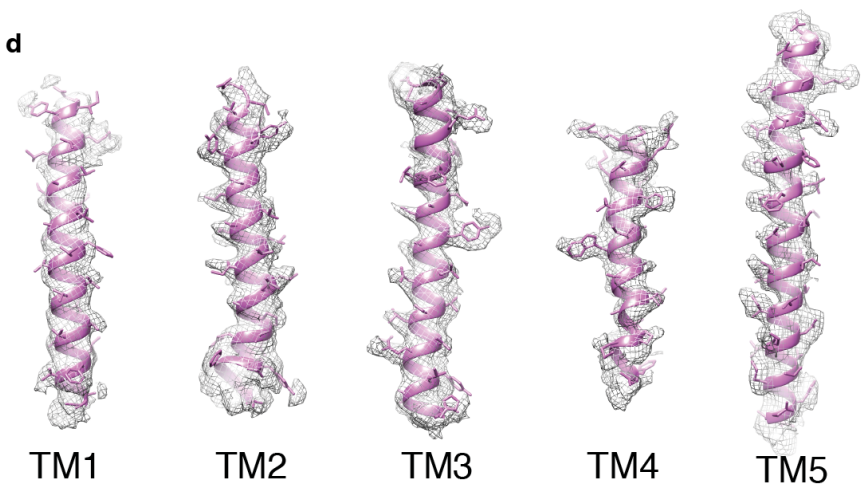

TM7

a5

$a N$

TM1

TM2

TM3

TM4
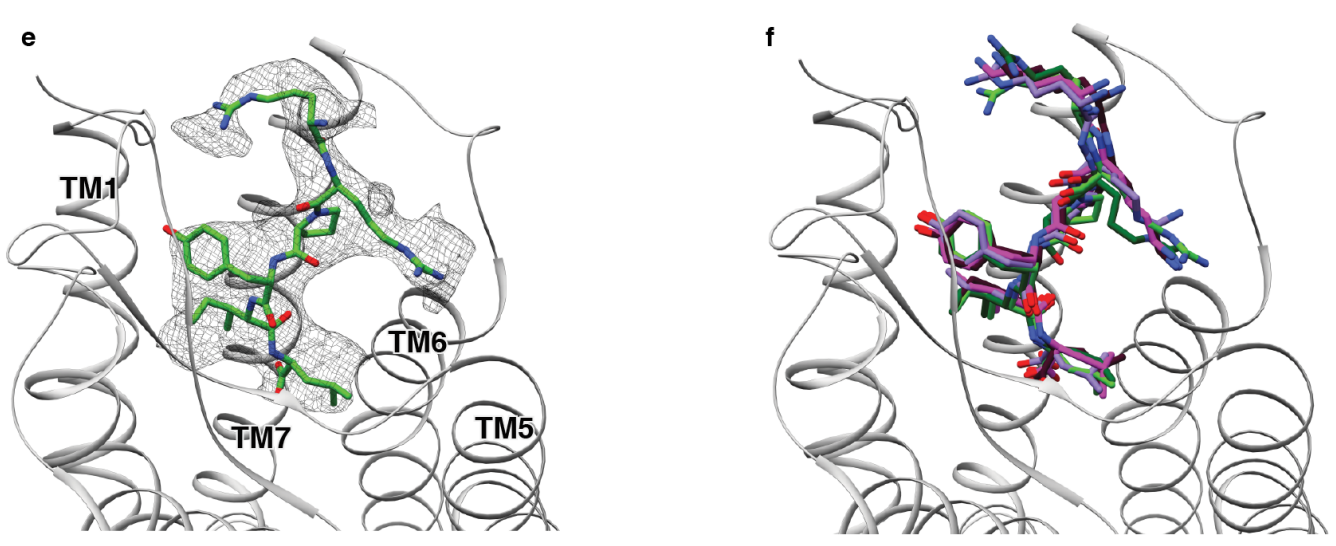


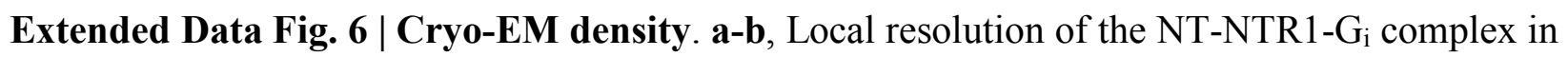
canonical state (a) and noncanonical state (b). The local resolution is calculated in Relion. c-d, Density and model for the transmembrane helices of NTR1 and the $\alpha 5$ and $\alpha \mathrm{N}$ helices of Go $\alpha_{\mathrm{i}}$ in the canonical state $(\mathbf{c})$ and noncanonical state $(\mathbf{d})$. e, Density and model for $\mathrm{NT}_{8-13 .} \mathbf{f}$, Superposition of the atomic models of $\mathrm{NT}_{8-13}$ from the NT-NTR1-G $\mathrm{G}_{\mathrm{i}} \mathrm{cND}$ complex in the canonical (light green), and noncanonical state (dark green) with NT from the NT-NTR1 crystal structure (purple; PDB 4XEE) and JMV449 (a NT analog) from the NTR1-Gi-micelle complex in the canonical (magenta; PDB 6OS9) and noncanonical state (dark red; PDB 6OSA). 
bioRxiv preprint doi: https://doi.org/10.1101/2020.06.11.145912; this version posted June 12, 2020. The copyright holder for this preprint (which was not certified by peer review) is the author/funder. All rights reserved. No reuse allowed without permission.
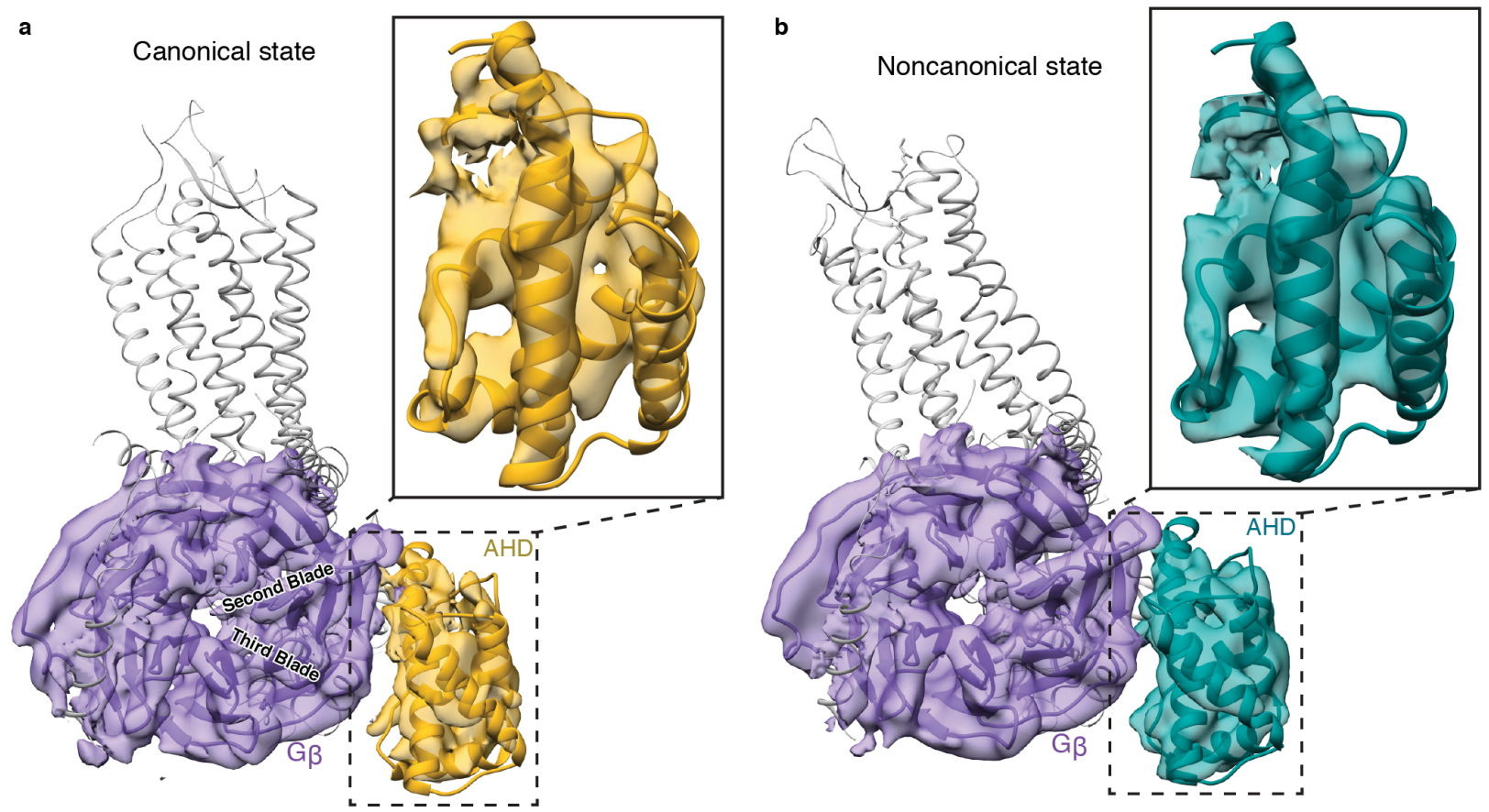

c

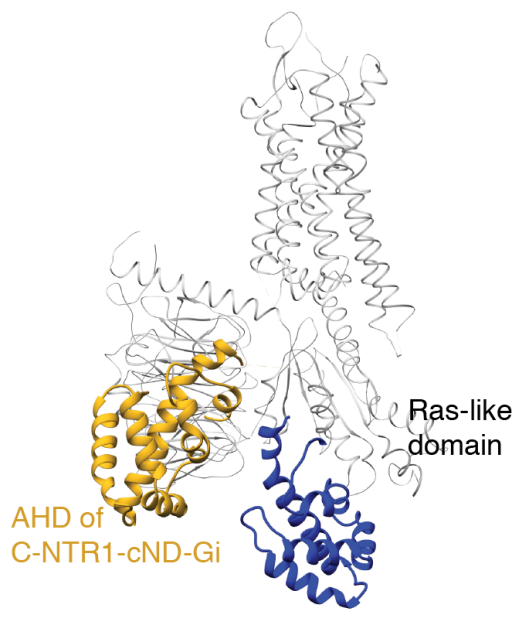

Crystal structure of Gi trimer with GDP

d

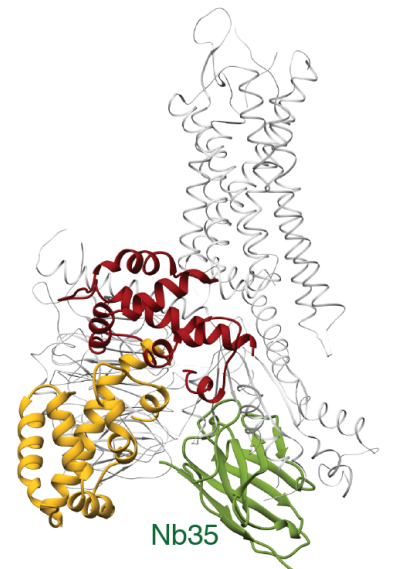

Crystal structure of $\beta 2 A R-G s$ with Nb35

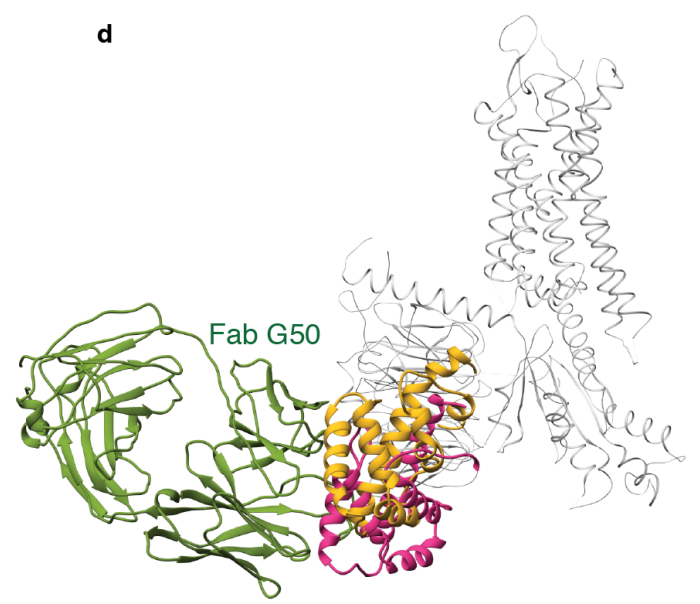

Cryo-EM structure of Rhodopsin-Gi with Fab G50

f

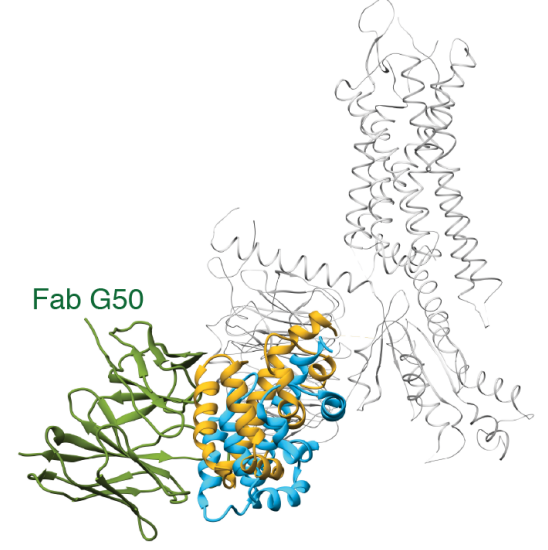

Cryo-EM structure of Smoothened-Gi with Fab G50 
Extended Data Fig. 7 | Structure of the $\alpha$-helical domain (AHD). a, Density maps and models showing the interaction between $\mathrm{G} \beta_{1}$ (purple) and $\mathrm{G} \alpha_{\mathrm{i} 1}$ AHD (gold) in the canonical state. Zoomin view of the G⿰ $\alpha_{i 1}$ AHD is shown. b, Density maps and models showing the interaction between $\mathrm{G} \beta_{1}$ (purple) and $\mathrm{G} \alpha_{\mathrm{i} 1}$ AHD (dark green) in the noncanonical state. Zoom-in view of the G $\alpha_{\mathrm{i} 1}$ AHD is shown. The models in (a) and (b) are superposed on the G $\beta_{1}$ subunits and are shown in the same view. AHD in both states interacts with the second and third blades of G $\beta_{1}$. c-f, Comparison of the AHD of the canonical state NT-NTR1-Gi-cND (gold) with c, A crystal structure of GDP-G $\mathrm{G}_{\mathrm{i}}$ (blue; PDB 1GP2), d, A crystal structure of $\beta_{2} \mathrm{AR}-\mathrm{G}_{\mathrm{s}}$ with nanobody Nb35 (AHD is dark red and Nb35 is green; PDB 3SN6), e, A cryo-EM structure of Rhodopsin- $\mathrm{G}_{\mathrm{i}}$ with Fab G50 (AHD is pink and Fab G50 is green; PDB 6CMO), and $\mathbf{f}$, A cryo-EM structure of Smoothened-G with Fab G50 (AHD is light blue and Fab G50 is green; PDB 6OT0). The models are superposed on the Ga Ras-like domain. 
a
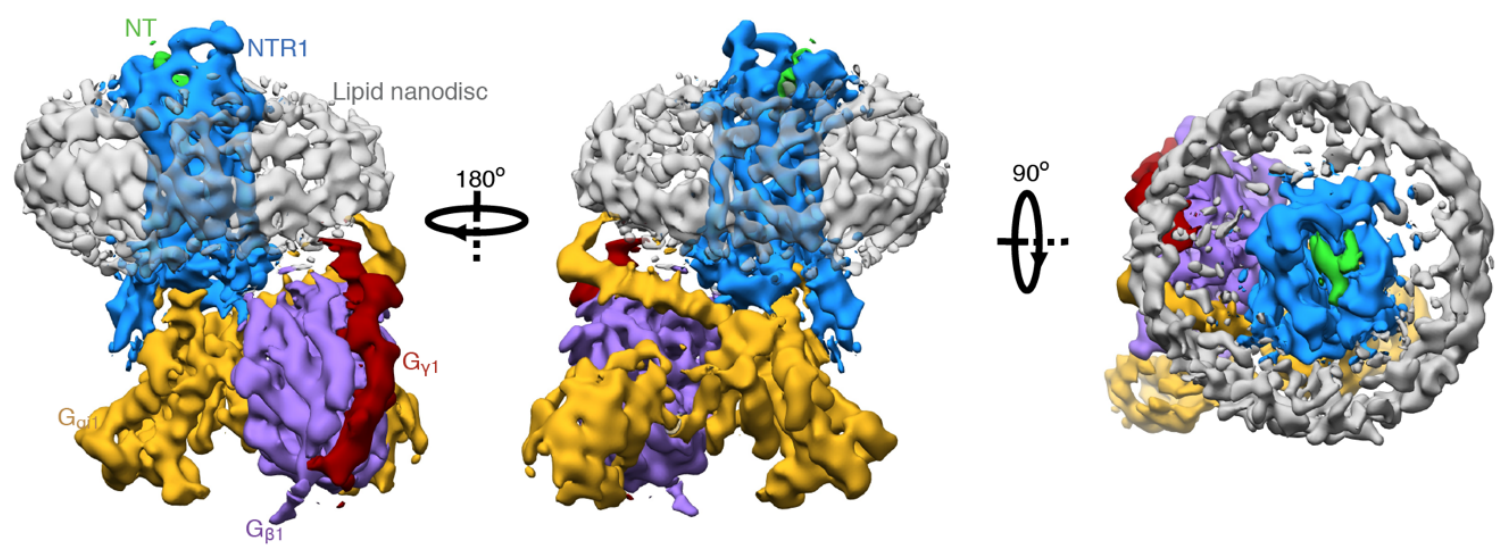

Canonical state

b
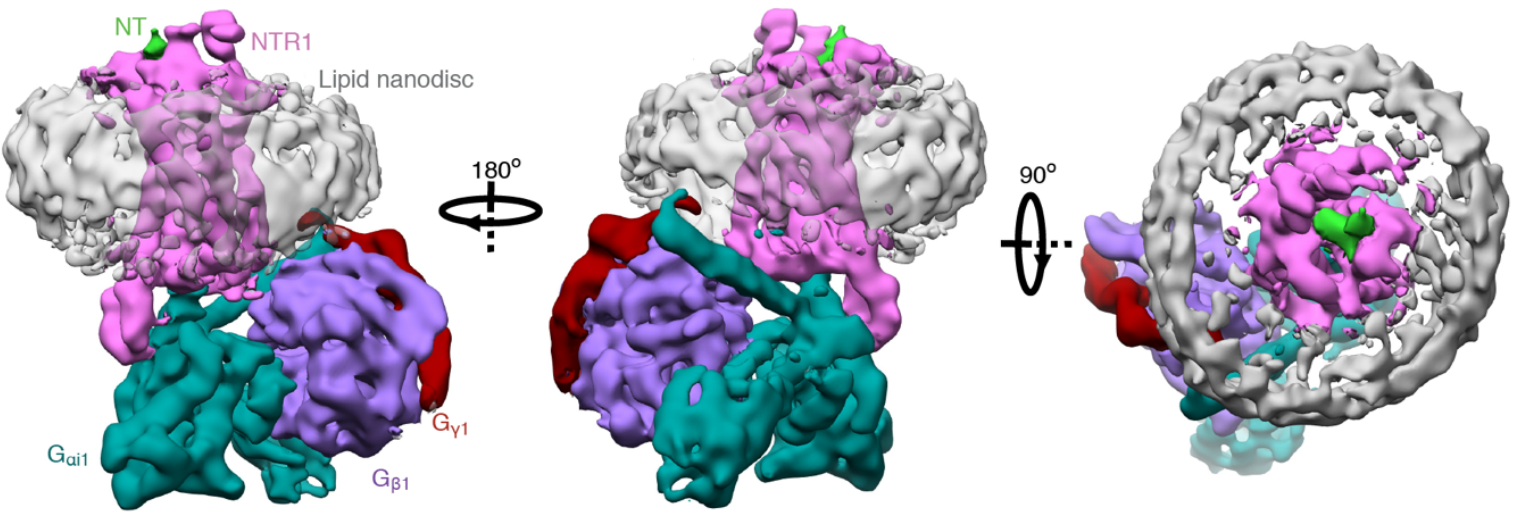

Noncanonical state
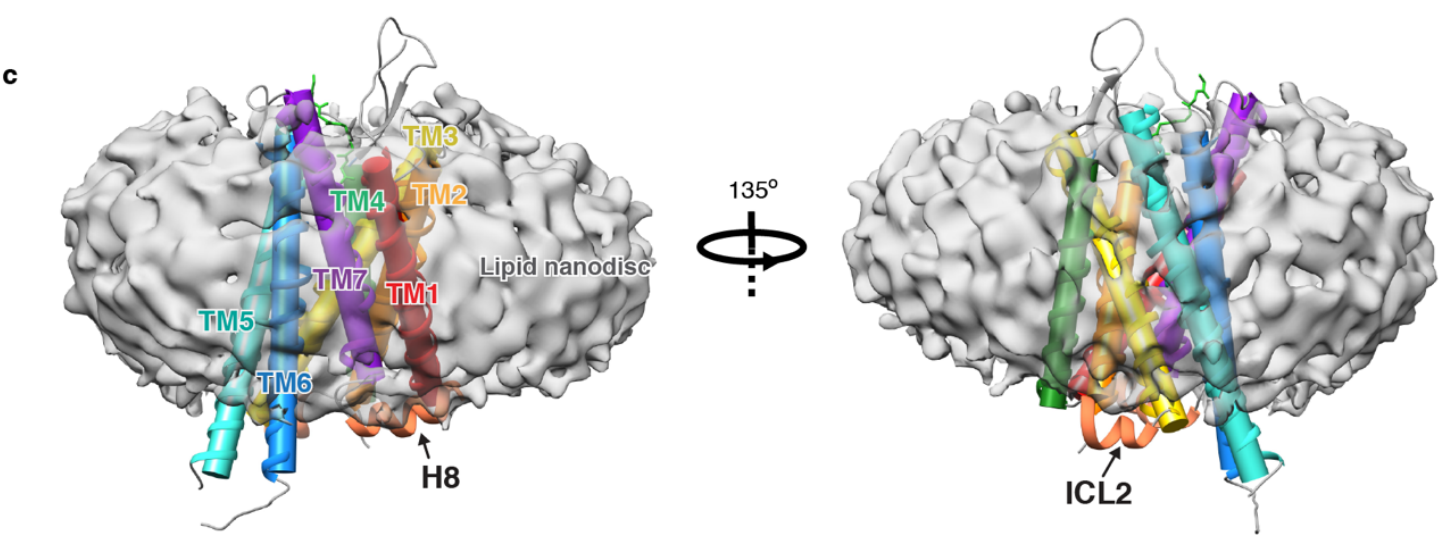

\section{Extended Data Fig. 8 | Cryo-EM structure of the NT-NTR1-G ${ }_{i}$ complex in lipid nanodiscs}

and the interaction with lipid. a, Three views of the cryo-EM density map of the NT-NTR1-G $\mathrm{i}^{-}$ cND complex in the canonical state. $\mathbf{b}$, Three views of the cryo-EM density map of the NT-NTR1$\mathrm{G}_{\mathrm{i}}-\mathrm{cND}$ complex in the noncanonical state. The maps in panels (a) and (b) are low-pass filtered to 
bioRxiv preprint doi: https://doi.org/10.1101/2020.06.11.145912; this version posted June 12, 2020. The copyright holder for this preprint (which was not certified by peer review) is the author/funder. All rights reserved. No reuse allowed without permission.

$5 \AA$ and colored by subunit. c, Two views of NT-NTR1 surrounded by nanodisc density. The transmembrane helices are shown in cylinder representation using the rainbow coloring scheme. ICL2 and helix H8 are partially submerged in lipid. 


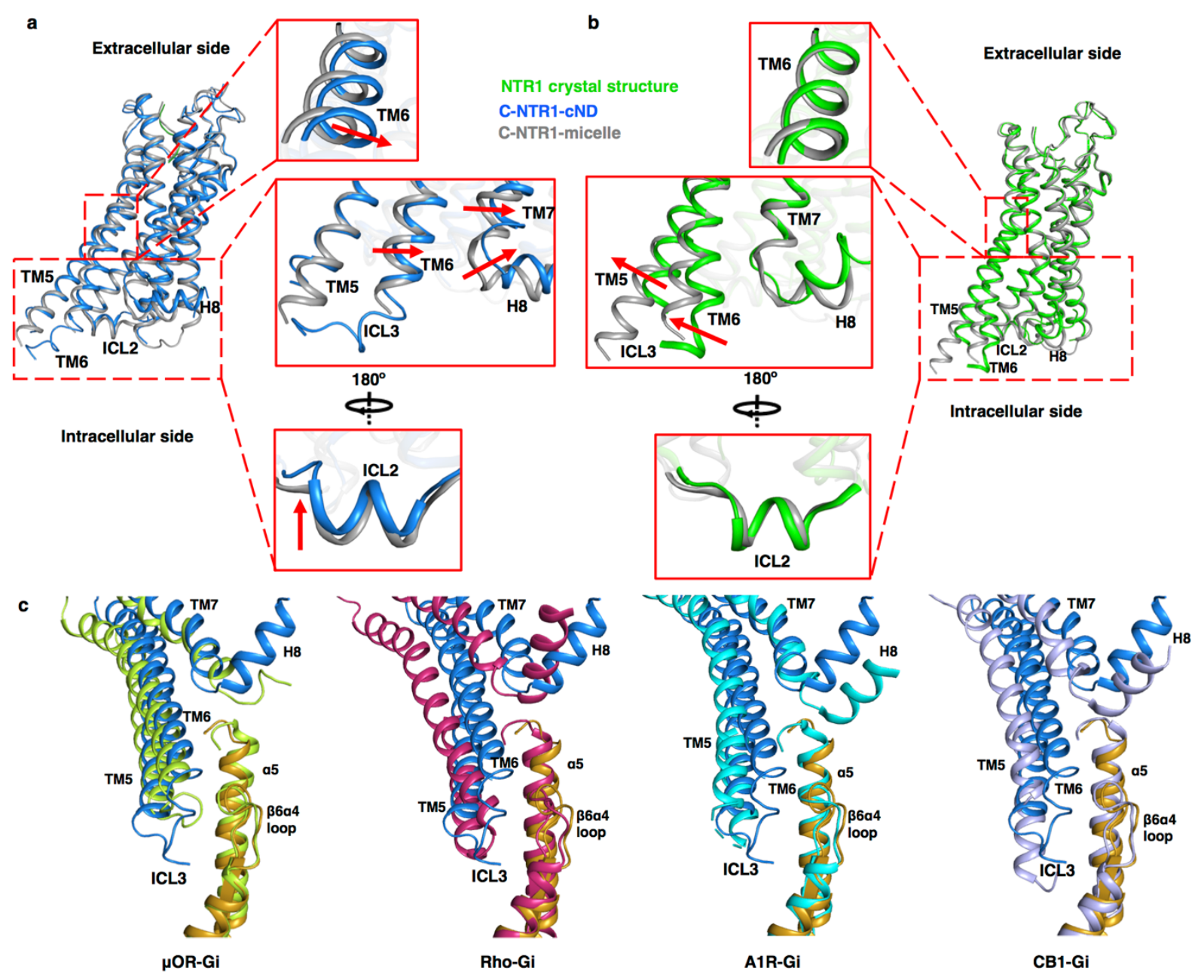

Extended Data Fig. 9 | Impact of lipid bilayer on the structure of NTR1. a, Comparison between the cryo-EM structures of the canonical states of NTR1 (with $\mathrm{G}_{\mathrm{i}}$ ) in lipid bilayer (blue) and detergent (gray). b, Structural comparison between the crystal structure of NTR1 in detergent (green) and the cryo-EM structure of the canonical state of NTR1 in complex with $\mathrm{G}_{\mathrm{i}}$ in detergent (gray). The atomic models in (a) and (b) are superposed on NTR1. c, Comparison of the

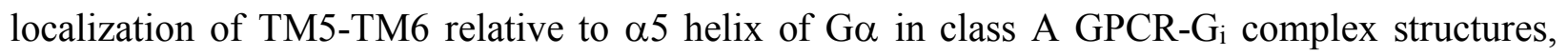
including the canonical state NTR1 (blue) in complex with $\mathrm{G}_{\mathrm{i}}$ (gold) structure reported in the current study, $\mu \mathrm{OR}-\mathrm{G}_{\mathrm{i}}$ (lime green; PDB 6DDE), Rho-G $\mathrm{G}_{\mathrm{i}}$ (hot pink; PDB 6CMO), A $\mathrm{A}_{1}-\mathrm{G}_{\mathrm{i}}$ (cyan; 
bioRxiv preprint doi: https://doi.org/10.1101/2020.06.11.145912; this version posted June 12, 2020. The copyright holder for this preprint (which was not certified by peer review) is the author/funder. All rights reserved. No reuse allowed without permission.

PDB 6D9H), and CB1-Gi (purple; PDB 6N4B). The models are superposed on the Ras-like domain of $\mathrm{G} \alpha$. 
a

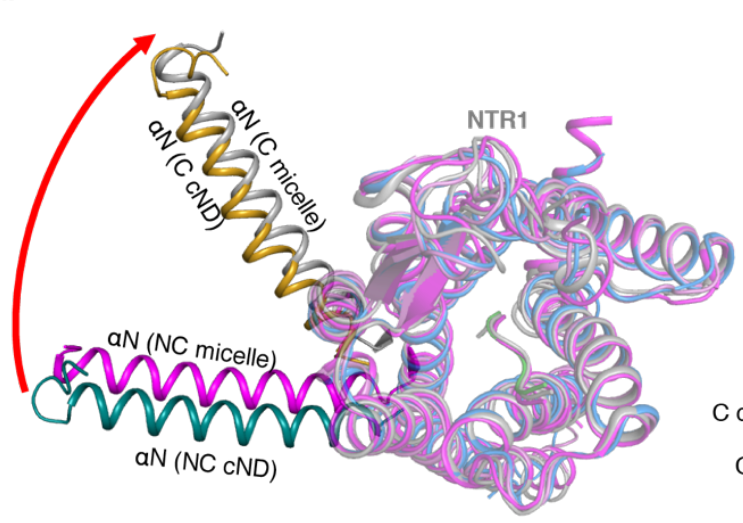

b

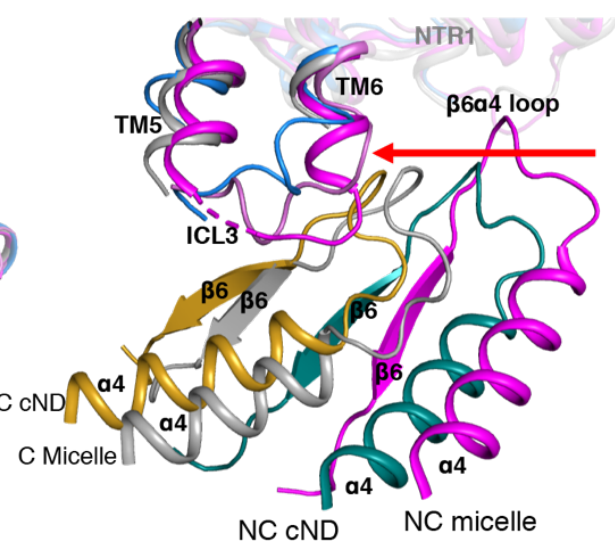

NC CND c

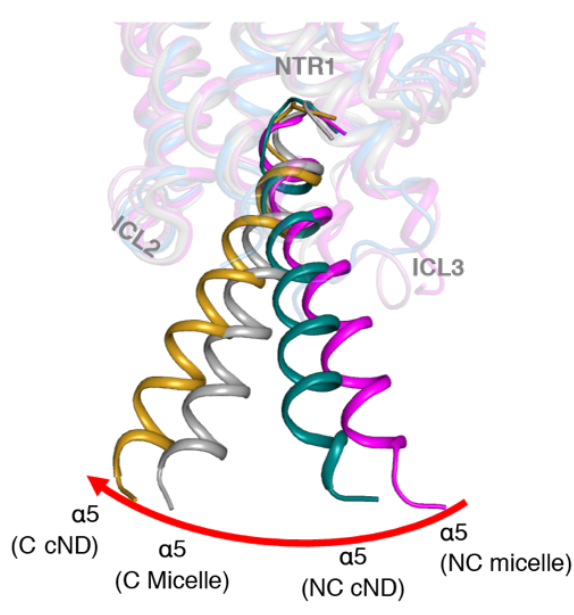

d

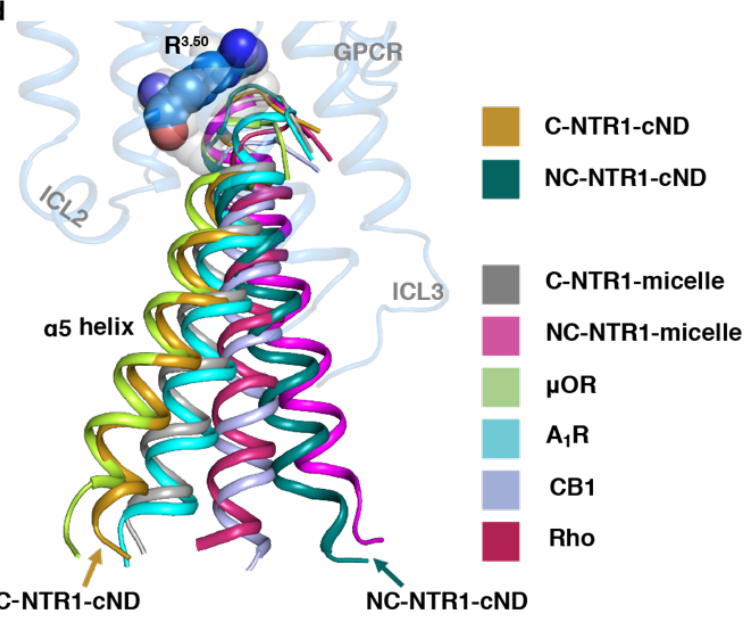

Extended Data Fig. 10 | Comparison of NTR1-G ${ }_{i}$ interaction in lipid bilayer with detergent

micelles. a-c, Superposed structure of C-state NTR1 (blue) and Ga (gold), NC-state NTR1 (orchid)

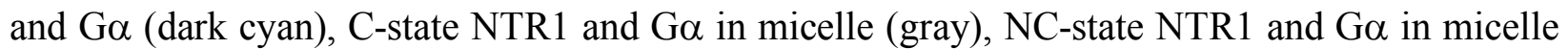
(magenta). The models are superposed on NTR1. a, extracellular view of NTR1 and $\alpha$ Nelix; $\mathbf{b}$, side view of NTR1 ICL3 and $\beta 6 \alpha 4$ loop; $\mathbf{c}$, side view of NTR1 and $\alpha 5$ helix. d, Comparison of the localization of $\alpha 5$ helix relative to GPCR in class A GPCR-Gi $\mathrm{G}_{\mathrm{i}}$ complex structures, including the canonical (gold) state and noncanonical (dark cyan) state structure reported in the current study, canonical (gray) and noncanonical (magenta) state of NTR1- $\mathrm{G}_{\mathrm{i}}$ in detergent micelle, $\mu \mathrm{OR}-\mathrm{G}_{\mathrm{i}}$, Rho$\mathrm{G}_{\mathrm{i}}, \mathrm{A}_{1} \mathrm{R}-\mathrm{G}_{\mathrm{i}}$, and $\mathrm{CB} 1-\mathrm{G}_{\mathrm{i}}$ in the same colors as panel (b). The structures are superposed on the 
bioRxiv preprint doi: https://doi.org/10.1101/2020.06.11.145912; this version posted June 12, 2020. The copyright holder for this preprint (which was not certified by peer review) is the author/funder. All rights reserved. No reuse allowed without permission.

GPCR. Residue $\mathrm{R}^{3.50}$ is shown as colored spheres in C-state NTR1 and as partially transparent gray spheres in the other GPCRs. 

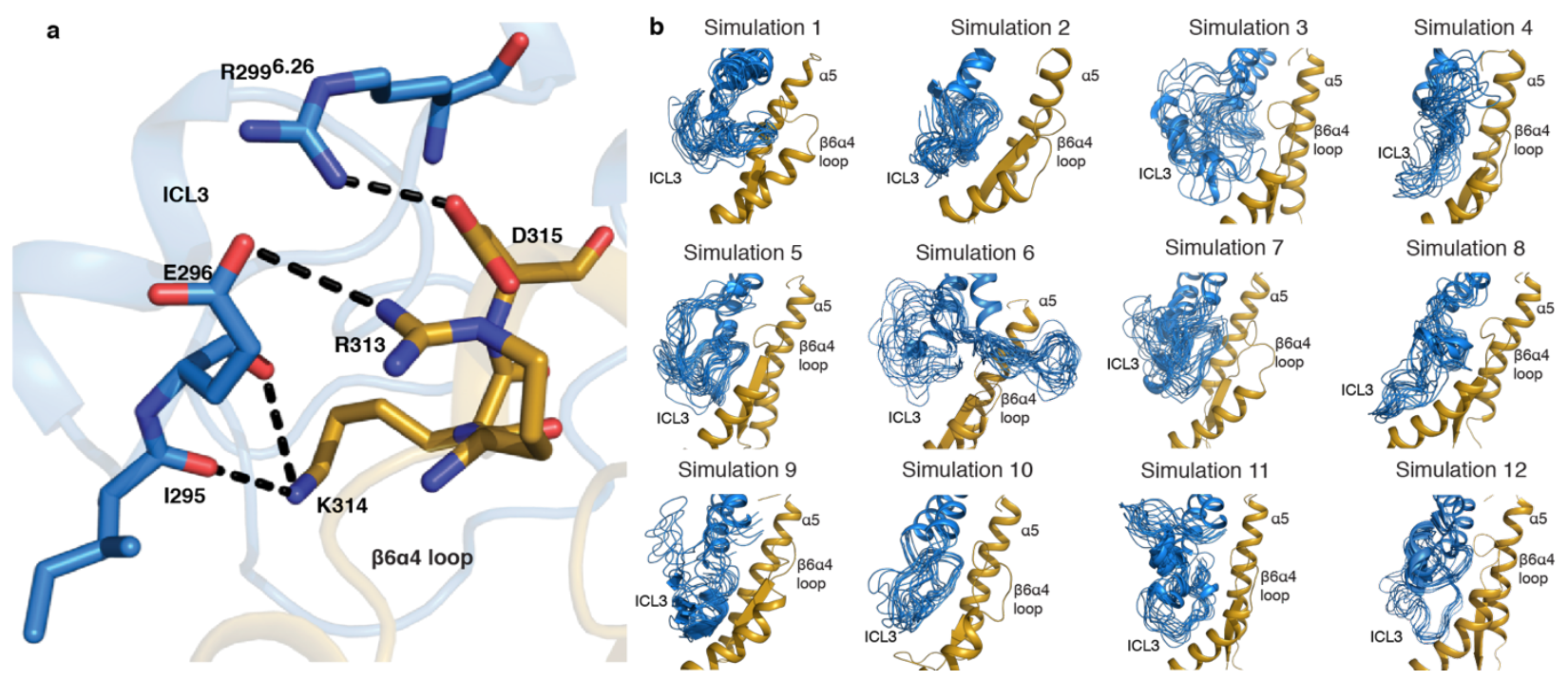

Extended Data Fig. 11 | Molecular dynamics (MD) simulation for the interaction between

ICL3 and the $\beta 6 \alpha 4$ loop. a, MD simulation showing salt bridges and hydrogen bonds form between TM6-ICL3 and $\beta 6 \alpha 4-1$ loop in the canonical state of NT-NTR1-Gi-cND represented by simulation 12. b, Dynamics of ICL3 for each independent simulation of the canonical state of NTNTR1-Gi-cND. Frames are sampled every $40 \mathrm{~ns}$ from each individual simulation. All 12 simulations show various interactions including salt bridges/hydrogen bonds between ICL3 and the $\beta 6 \alpha 4$-loop. An example of detailed interactions is shown in (a). NTR1 is colored in blue and $\mathrm{G}_{\mathrm{i}}$ in gold in (a-b). 
Extended Data Table 1. Cryo-EM data collection, refinement and validation statistics

\begin{tabular}{|c|c|c|}
\hline & $\begin{array}{l}\text { NT-NTR1-G } \mathrm{G}_{\mathrm{i}} \mathrm{c} \mathrm{ND} \\
\text { Canonical state } \\
(\text { EMDB-xxxx) } \\
(\mathrm{PDB} \times \mathrm{xxx})\end{array}$ & $\begin{array}{l}\text { NT-NTR1-G } \mathrm{G}_{\mathrm{i}} \mathrm{cND} \\
\text { Noncanonical state } \\
\text { (EMDB-xxxx) } \\
(\mathrm{PDB} \times \mathrm{xxx})\end{array}$ \\
\hline \multicolumn{3}{|l|}{ Data collection and processing } \\
\hline Magnification & 105,000 & 105,000 \\
\hline Voltage (kV) & 300 & 300 \\
\hline Electron exposure $\left(\mathrm{e}^{-} / \AA^{2}\right)$ & 57 & 57 \\
\hline Defocus range $(\mu \mathrm{m})$ & -1.2 to -2.5 & -1.2 to -2.5 \\
\hline Pixel size $(\AA)$ & 0.825 & 0.825 \\
\hline Symmetry imposed & $\mathrm{C} 1$ & $\mathrm{C} 1$ \\
\hline Initial particle images (no.) & $4,367,542$ & $4,367,542$ \\
\hline Final particle images (no.) & 575,791 & 324,002 \\
\hline Map resolution $(\AA)$ & 4.1 & 4.2 \\
\hline FSC threshold & 0.143 & 0.143 \\
\hline \multicolumn{3}{|l|}{ Refinement } \\
\hline Refinement software & \multicolumn{2}{|c|}{ Coot 0.9-pre, Phenix-dev-3318 } \\
\hline Initial model used (PDB code) & \multicolumn{2}{|c|}{ 4BUO, $1 \mathrm{GP} 2,6 \mathrm{OY} 9$} \\
\hline Resolution limit set in refinement $(\AA)$ & 4.1 & 4.2 \\
\hline Map sharpening $B$ factor $\left(\AA^{2}\right)$ & -245 & -204 \\
\hline \multicolumn{3}{|l|}{ Model composition } \\
\hline Non-hydrogen atoms & 6959 & 6979 \\
\hline Protein residues & 883 (6901 atoms) & 882 (6921 atoms) \\
\hline Ligands & 1 (6 residues, 58 atoms) & 1 (6 residues, 58 atoms) \\
\hline \multicolumn{3}{|l|}{$B$ factors $\left(\AA^{2}\right)$} \\
\hline Protein & 73.57 & 73.56 \\
\hline Ligand & 66.76 & 66.76 \\
\hline \multicolumn{3}{|l|}{ R.m.s. deviations } \\
\hline Bond lengths $(\AA)$ & 0.006 & 0.007 \\
\hline Bond angles $\left({ }^{\circ}\right)$ & 1.052 & 1.365 \\
\hline \multicolumn{3}{|l|}{ Validation } \\
\hline MolProbity score & 1.90 & 1.96 \\
\hline Clashscore & 8.36 & 9.77 \\
\hline EMRinger score & 1.46 & 1.17 \\
\hline Poor rotamers $(\%)$ & 0.53 & 1.05 \\
\hline \multicolumn{3}{|l|}{ Ramachandran plot } \\
\hline Favored (\%) & 93.11 & 93.33 \\
\hline Allowed (\%) & 6.89 & 6.67 \\
\hline Disallowed (\%) & 0 & 0 \\
\hline
\end{tabular}

